\title{
Electromagnetic Wave Reflection From an Oscillating, Collision-Free Magneto-Ionic Medium
}

\author{
O. E. H. Rydbeck \\ Research Laboratory of Electronics, Chalmers University of Technology, Gothenburg, Sw€den
}

(Received May 19, 1964; revised August 21, 1964)

\begin{abstract}
A collision-free or low collision magneto-ionic medium is easily perturbed by a driving or pumping wave, even of moderate power. Unless the pump wave has longitudinal propagation, it will generate longitudinal electron velocities as well as differential space charge densities. These quantities, as well as the transverse, pump field electron velocities, will affect the propagation of any (low power) signal or probing wave propagating through the medium, which now has oscillating and traveling electron velocity and electron density ripples.

If the pump wave has an angular frequency $\omega_{p}$, and that of the signal wave is $\omega_{s}$, two first order sum and difference frequency $\left(\omega_{ \pm 1}=\omega_{p} \pm \omega_{s}\right)$ waves are generated in the medium. It is shown that the generation of these waves, for which specific refraction laws hold, is greatly enhanced if a parametric traveling wave resonance develops in the system or if the nonlinear driving force experiences a local resonance. Under certain conditions, sum and/or difference frequency waves, usually with two kinds of polarization, will radiate away from the resonance interaction region. This generation is dealt with in detail for homogeneous media, with a discontinuous boundary, and for slowly inhomogeneous ones.

Waves of this kind should be generated easily by a topside sounder of moderate power. It should also be possible, with present day techniques, to record sum and difference frequency "echoes" at the ground, if a very powerful pump wave transmitter were used. It is interesting to note that not only the true height of normal reflection (at $\omega_{e}=\omega_{s}$, where $\omega_{e}$ is the angular plasma frequency) but also the electron density gradient, at the same level, should be obtainable by such measurements, provided $\omega_{p} \gg \omega_{s}$. Since $\omega_{p}$ could be left unchanged, while $\omega_{s}$ is swept through the sounding range of interest, the technical arrangements for such experiments, which might be very rewarding, should not be overly complicated.
\end{abstract}

\section{Electromagnetic Wave Reflection From the Oscillating, Isotropic, Ionized Medium}

We assume that the signal - or probing-wave, of angular frequency $\omega_{s}$, and the high power "pump" wave, have polarizations and angles of incidence upon the ionized as shown in figure 1.

The pump wave generates a nonlinear variation, $\Delta N$, of the mean electron density $N_{e}$, and a longitudinal electron velocity, $v_{L}$, in the direction of the pump wave normal. These electron density and velocity "fluctuations" travel through the medium, locked to the high power wave, and can be written as follows [Rydbeck, 1961],

$$
\begin{aligned}
\Delta N & =\gamma N_{e} \cos \left(\omega_{p} t-\bar{k}_{p} \bar{r}\right), \\
v_{L} & =v_{L}^{0} \cos \left(\omega_{p} t-\bar{k}_{p} \bar{r}\right),
\end{aligned}
$$

where $\omega_{p}$ and $\bar{k}_{p}$ are equal to twice the corresponding parameters of the linear pump wave. $\gamma$ and $v_{L}^{0}$ are proportional to the square of its amplitude.

Besides this nonlinear "plasma wave," (1.1) and (1.2), one must also consider the linear transverse electron velocities, $v_{T, x}$ and $v_{T, z}$, of the pump wave. The total electron velocities associated with the pump wave thus become

$$
\begin{gathered}
v_{p, x}=v_{T, x}+v_{L, x}=v_{T} \cos \varphi_{\mathrm{II}, p}+v_{L} \sin \varphi_{\mathrm{II}, p}, \\
v_{p, z}=v_{T, z}+v_{L, z}=-v_{T} \sin \varphi_{\mathrm{II}, p}+v_{L} \cos \varphi_{\mathrm{II}, p} .
\end{gathered}
$$




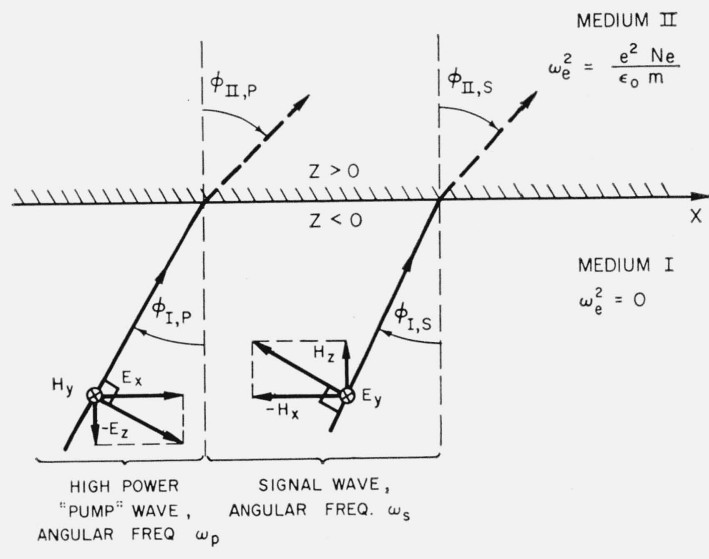

Figure 1. Depicting polarizations and angles of incidence of signal and pump waves.

\subsection{Only Electron Density Oscillations Considered}

In order to distinguish between the two nonlinear effects, we first study the reflection property of the ionized medium when the velocity fluctuations are neglected, i.e., we assume $v_{p}=0=v_{T}$, and disregard, for the moment, the fact that these velocities, in reality, always differ from zero when $\Delta N$ does so.

The wave equation describing the propagation of the signal wave now can be written [Rydbeck, 1963],

$$
\left[\frac{\partial^{2}}{\partial z^{2}}+\frac{\partial^{2}}{\partial x^{2}}-\frac{1}{c_{0}^{2}} \frac{\partial^{2}}{\partial t^{2}}-\frac{\omega_{e}^{2}}{c_{0}^{2}}\left\{1+\gamma \cos \left(\omega_{p} t-\bar{k}_{p} \bar{r}\right)\right\}\right] \psi=0
$$

where $\psi$ is proportional to $v_{y}$ (the electron velocity of the signal wave) and is related to $E_{y}$ in the following (arbitrarily) normalized manner

$$
E_{y}=\frac{1}{j \omega_{s}} \frac{\partial \psi}{\partial t}
$$

$c_{0}=1 / \sqrt{\mu_{0} \epsilon_{0}}=$ the electromagnetic wave velocity in vacuum, and

$$
\omega_{e}=\sqrt{\frac{e^{2} N_{e}}{m \epsilon_{0}}}
$$

is the mean electronic plasma frequency.

We introduce (see also fig. 1)

and

$$
\left.\begin{array}{rl}
k_{p} & =\frac{\omega_{p}}{c_{0}} n_{p} ; k_{p, x}=\frac{\omega_{p}}{c_{0}} n_{p, x}=k_{p} \sin \varphi_{\mathrm{II}, p}, \\
k_{p, z} & =\frac{\omega_{p}}{c_{0}} n_{p, z}=k_{p} \cos \varphi_{\mathrm{II}, p},
\end{array}\right\}
$$

where $n_{p}$ is assumed to be a known function of the angular pump frequency, $\omega_{p}$. In order to make our results more general we do not specify the dispersion relation determining $k_{p}$, since one can, in principle, think of other types of pump waves besides electromagnetic ones.

Next we introduce the variable

$$
u=\frac{1}{2}\left(\omega_{p} t-\bar{k}_{p} \bar{r}\right)
$$


and assume that $\psi$ can be written as follows, viz,

where

$$
\psi=\psi_{q}=e^{j\left(\omega_{q} t-\bar{k}_{q} \bar{r}\right)} \Pi_{x}(u)
$$

$$
k_{q}=\frac{\omega_{q}}{c_{0}} \sqrt{1-\frac{\omega_{e}^{2}}{\omega_{q}^{2}}}=\frac{\omega_{q}}{c_{0}} n_{q}=k_{0, q} n_{q}
$$

and $\omega_{q}$ denotes the angular frequency of an unperturbed wave, i.e., for $\gamma=0$. When $\Pi_{q}(u)$ reduces to a constant, as it will in absence of the pump wave, (1.9) represents a plane, stationary monochromatic wave.

Now, let us introduce the characteristic parameter

where

$$
\theta_{0, q}=\frac{4 \omega_{q}^{2}}{\omega_{p}^{2}}\left(\frac{1-d_{q}}{1-n_{p}^{2}}\right)^{2}
$$

$$
d_{q}=n_{q, x} n_{p, x}+n_{q, z} n_{p, 2}=n_{q} n_{p} \cos \left(\varphi_{\mathrm{II}, p}-\varphi_{\mathrm{II}, q}\right) .
$$

$\Pi_{q}$ can now be written

$$
\Pi_{q}=e^{-j \sqrt{\theta_{0, q}} u} \pi_{q}^{(2)},
$$

where $\Pi_{q}^{(2)}$ is a proper solution of the Mathieu equation

$$
\left(\frac{d^{2}}{d u^{2}}+\theta_{0, q}+2 \theta_{1} \cos 2 u\right) \Pi_{q}^{(2)}=0
$$

and

$$
\theta_{1}=\gamma \frac{2 \omega_{e}^{2}}{\omega_{p}^{2}} \frac{1}{1-n_{p}^{2}}
$$

It is interesting to notice that the important parameter $\theta_{1}$ is independent of $\omega_{q}$ (and $k_{q}$ ). The solution of (1.14) is now for our purposes written

$$
\Pi_{q}^{(2)}=\beta_{q} e^{\mu} q^{u} \sum_{n=-\infty}^{n=+\infty} \alpha_{q, n} e^{j 2 n u}
$$

where $\mu_{q}$ is the proper root of Hill's determinantal equation, ${ }^{1}$

$$
\sin ^{2}\left(j \frac{\pi}{2} \mu_{q}\right)=\Delta(0) \sin ^{2}\left(\frac{\pi}{2} \sqrt{\theta_{0, q}}\right)
$$

and the amplitude coefficients can be obtained from the relations

$$
\left\{\left(\mu_{q}+j 2 n\right)^{2}+\theta_{0, q}\right\} \alpha_{q, n}+\theta_{1}\left(\alpha_{q, n+1}+\alpha_{q, n-1}\right)=0 \quad(n=\ldots,-2,-1,0,+1,+2, \ldots) .
$$

As is well known from the theory of Mathieu equations, instability regions are centered around the $\theta_{0, q}$ values $1,4,9$, etc. In this connection we are mainly interested in the first, and most important one $\left(\theta_{0, q}=1\right)$, since we will neglect the formation of higher order "side bands" $\omega=\omega_{q} \pm m \omega_{p},(m=2,3, \ldots)$. When this is the case, and if $\theta_{1}^{2}<<1$, we can write [Rydbeck, $1954]$

$$
\Delta(0)=1+2 \frac{28}{90} \theta_{1}^{2}+0\left(\theta_{1}^{4}\right) \quad\left(\theta_{1}^{2}<<1 ; \theta_{0, q} \approx 1\right) .
$$

According to (1.17) $\left|j \frac{\pi}{2} \mu_{q}\right|$ does not differ much from $\left|\frac{\pi}{2} \sqrt{\theta_{0, q}}\right|$. 
When $\theta_{1}=0$, i.e., for an unpumped medium, we have

$$
\mu_{q}= \pm j \sqrt{\theta_{0, q}} \quad\left(\theta_{1}=0\right) .
$$

Since $\psi_{q}$ must reduce to $\exp \left\{j\left(\omega_{q} t-\bar{k}_{q} \bar{r}\right)\right\}$ in this case (no waves are assumed to come from $z=+\infty)$, we must according to (1.13) use the upper sign in (1.20). For the moderately pumped medium we therefore write

and obtain

$$
\mu_{q}=j\left(\sqrt{\theta_{0, q}}+b_{q}\right), \quad\left(b_{q} \rightarrow 0, \text { if } \theta_{1} \rightarrow 0\right)
$$

$$
\psi_{q}=e^{j\left(\omega_{q} t-\bar{k} \bar{r}^{\bar{r}}+j b_{q} u\right.} \beta_{q} \sum_{n=-\infty}^{n=+\infty} \alpha_{q, n} e^{j 2 n u} .
$$

If we introduce (1.21) in (1.17), we obtain

$$
\sin \left(\frac{\pi}{2} b_{q}\right)=\sin a_{q}\left\{\sqrt{\Delta(0)} \cos a_{q}-\sqrt{1-\Delta(0) \sin ^{2} a_{q}}\right\},
$$

where

$$
a_{q}=\frac{\pi}{2} \sqrt{\theta_{0 . q}}
$$

With the notation

$$
\Delta(0)=1+2 \xi
$$

where $\xi^{2}<<1$, we obtain the following approximate "bridging" solution of (1.23),

$$
b_{q} \approx-\frac{1}{2}\left\{\theta_{0, q}-1-\sqrt{\left(\theta_{0, q}-1\right)^{2}-\frac{32}{\pi^{2}} \xi}\right\}, \quad\left(\xi^{2}<<1\right)
$$

where it is to be noted that, by (1.19),

$$
\frac{32}{\pi^{2}} \xi=\frac{32}{\pi^{2}} \frac{28}{90} \theta_{1}^{2}=1.01 \theta_{1}^{2} \cong \theta_{1}^{2} .
$$

The instability zones thus are determined by the relations

$$
\left.\begin{array}{l}
-\frac{\theta_{1}}{2} \leq \sqrt{\theta_{0, q}}-1 \leq+\frac{\theta_{1}}{2} \quad\left(\sqrt{\theta_{0, q}} \approx 1\right) \\
-\frac{\theta_{1}}{2} \leq \sqrt{\theta_{0, q}}+1 \leq+\frac{\theta_{1}}{2} \quad\left(\sqrt{\theta_{0, q}} \approx-1\right) .
\end{array}\right\}
$$

When $\theta_{0, q}=1, b_{q}= \pm j \frac{\theta_{1}}{2},\left(\xi^{2}<<1\right)$. In the center of the instability regions the waves thus vary in amplitudes as

$$
e^{ \pm \frac{\gamma}{2} \frac{\omega_{e}^{2}}{\omega_{p}^{2}} \frac{1}{1-n_{p}^{2}}\left(\omega_{p} t-\bar{k}_{p} \bar{r}\right)}
$$

or, if $1-n_{p}^{2}=\omega_{e}^{2} / \omega_{p}^{2}$, like $\exp \left\{ \pm \frac{\gamma}{2}\left(\omega_{p} t-\bar{k}_{p} \bar{r}\right)\right\}$. It is interesting to note that this growth or decay is independent of $\omega_{q}$ (and $k_{q}$ ). As "viewed" by the traveling pump wave, the amplitudes of all other waves in the system do not appear to change. It depends upon the boundary condition whether one gets growing or evanescent waves (with time), or both. In our particular case, where no waves are assumed to come from $z=+\infty$, the unstable waves grow with time and decay with $\bar{k}_{p} \bar{r}$. In directions transverse to $\bar{k}_{p}$, the amplitudes change with time only.

By (1.18) the amplitude coefficient relations can be given the following practical forms.

$$
\left(n+b_{q} / 2\right)\left(\sqrt{\theta_{0, q}}+n+b_{q} / 2\right) \alpha_{q, n}=\frac{\theta_{1}}{4}\left(\alpha_{q, n-1}+\alpha_{q, n+1}\right) \quad(n=\ldots,-2,-1,0,+1,+2, \ldots) .
$$


If we neglect the $\alpha_{q, \pm 2}$ terms, $\alpha_{q,+1}$, and $\alpha_{q_{v}-1}$ become

$$
\begin{gathered}
\alpha_{q,+1} \simeq \frac{\theta_{1}}{4} \frac{\alpha_{q, 0}}{\left(\sqrt{\theta_{0, q}}+1+b_{q} / 2\right)\left(1+b_{q} / 2\right)} \\
\alpha_{q,-1} \simeq-\frac{\theta_{1}}{4} \frac{\alpha_{q, 0}}{\left(\sqrt{\theta_{0, q}}-1+b_{q} / 2\right)\left(1-b_{q} / 2\right)} .
\end{gathered}
$$

If we make use of (1.25) these relations can be given the following final forms

$$
\begin{aligned}
\alpha_{q,+1} & \simeq \frac{\theta_{1}}{2} \frac{\alpha_{q, 0}}{\left\{\sqrt{\theta_{0, q}}+1+\sqrt{\left(\sqrt{\theta_{0, q}}+1\right)^{2}-\left(-\theta_{1} / 2\right)^{2}}\right\}} \\
\alpha_{q,-1} & \simeq-\frac{\theta_{1}}{2} \frac{\alpha_{q, 0}}{\left\{\sqrt{\theta_{0, q}}-1+\sqrt{\left(\sqrt{\theta_{0, q}}+1\right)^{2}-\theta_{1}^{2} / 4}\right\}},
\end{aligned}
$$

from which it appears that $\left|\alpha_{q,+1}\right|$ and $\left|\alpha_{q,-1}\right|$ are equal to $\alpha_{q, 0}$ at the boundaries of the instability zones. At the center of these zones $\alpha_{q,+1}= \pm j \alpha_{q, 0}$, where the upper sign corresponds to growing waves (with time), and $\alpha_{q,-1}= \pm j \alpha_{q, 0}$.

From (1.18) it follows that higher order coefficients experience resonances (and the associated mixed frequency field instabilities), when $\sqrt{\theta_{0, q}}= \pm 2, \pm 3$, etc.

Next let us introduce the following notations, viz,

$$
\left.\begin{array}{l}
q=0: \omega_{0} \rightarrow \omega_{s} \\
q=+1: \omega_{+1} \rightarrow \omega_{s}+\omega_{p} \\
q=-1: \omega_{-1} \rightarrow \omega_{s}-\omega_{p}
\end{array}\right\} \text { if } \theta_{1} \rightarrow 0
$$

The corresponding $\psi$ functions become:

$$
\begin{aligned}
& \psi_{0}=e^{+j\left\{\left(\omega_{0}+b_{0} \omega_{p} / 2\right)\right.} t^{\left.-\left(\bar{k}_{0}+b_{0} \bar{k}_{p} / 2\right) \bar{r}\right\}} \beta_{0}\left\{\alpha_{0,0}+\alpha_{0,+1} e^{+j\left(\omega_{p} t-\bar{k}_{p} \bar{r}\right)}+\alpha_{0,-1} e^{-j\left(\omega_{p} t-\bar{k}_{p} \bar{r}^{r}\right)}\right. \\
& \left.\left.+\alpha_{0,+2} e^{+j 2\left(\omega_{p} t-\bar{k}\right.} p^{\bar{r}}\right)+\alpha_{0,-2} e^{-j 2\left(\omega_{p} t-\bar{k} p^{r}\right)}+\ldots\right\},
\end{aligned}
$$

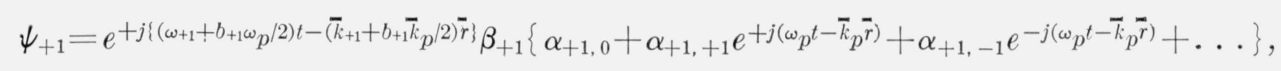

$$
\begin{aligned}
& \psi_{-1}=e^{+j\left\{\left(\omega_{-1}+b-1 \omega_{p} / 2\right) t-\left(\bar{k}_{-1}+b_{-1} \bar{k}_{p} / 2\right) \bar{r}\right.} \beta_{-1}\left\{\alpha_{-1,0}+\alpha_{-1,+1} e^{+j\left(\omega_{p} t-\bar{k}_{p} \bar{r}\right)}+\alpha_{-1,-1} e^{-j\left(\omega_{p} t-\bar{k}_{p} \bar{r}\right)}+\ldots\right\} \cdot
\end{aligned}
$$

Similar expressions can be written down for $q=+2\left(\omega_{+2} \rightarrow \omega_{s}+2 \omega_{p} ; \theta_{1} \rightarrow 0\right), q=-2\left(\omega_{-2} \rightarrow \omega_{s}-2 \omega_{p}\right.$; $\left.\theta_{1} \rightarrow 0\right)$, etc. The total field is

$$
\psi_{t o t}=\sum_{q} \psi_{q}(q=\ldots,-2,-1,0,+1,+2, \ldots) .
$$

The frequencies $\omega_{q}$ and the amplitude coefficients $\beta_{q}$ are determined by the boundary conditions, and so are $k_{q, x}$. Thus, if the incident signal wave has an angular frequency $\omega_{s}$, and an angle of incidence $\varphi_{I, s}$, one obtains

$$
\begin{gathered}
\omega_{0}+b_{0} \omega_{p} / 2=\omega_{s}, \\
k_{0, x}+b_{0} k_{p, x} / 2=k_{s, x}=\frac{\omega_{s}}{c_{0}} \sin \varphi_{I, s}=k_{0, s} \sin \varphi_{I, s},
\end{gathered}
$$

relations that can be satisfied only when $\operatorname{Im}\left(b_{0}\right)=0$, i.e., outside the instability zones.

Thus, according to (1.31), there are waves of frequencies $\omega_{s}, \omega_{s} \pm \omega_{p}, \omega_{s} \pm 2 \omega_{p}$, etc., present in the system. Therefore (1.33) and (1.34) yield

$$
\omega_{ \pm 1}+b_{ \pm 1} \omega_{p} / 2=\omega_{s} \pm \omega_{p}
$$




$$
k_{ \pm 1, x}+b_{ \pm 1} k_{p, x} / 2=k_{s, x} \pm k_{p, x}
$$

which can only be satisfied when $\operatorname{Im}\left(b_{ \pm 1}\right)=0$.

In the following we will neglect the instability zones, which will be discussed in more detail in sec. 1.3 in connection with a study of the self oscillation properties of the pumped medium.

If we neglect the instability zones, we also neglect $b_{q}$, which is very small outside the same (if $\xi^{2}<<1$ ). Relations $(1.35)$ et seq. now reduce to

$$
\omega_{0}=\omega_{s} ; \omega_{ \pm 1}=\omega_{s} \pm \omega_{p} ; k_{0, x}=k_{s, x} ; k_{ \pm 1, x}=k_{s, x} \pm k_{p, x}\left(b_{q}=0\right)
$$

These relations yield the reflection laws for the sum and difference frequency waves

$$
\sin \varphi_{I, \pm 1}=\frac{1}{\omega_{s} \pm \omega_{p}}\left(\omega_{s} \sin \varphi_{I, s} \pm \omega_{p} \sin \varphi_{\mathrm{I}, p}\right)
$$

If we include also the higher order waves this relation assumes the general form [Rydbeck 1962]

$$
\sin \varphi_{I, n}=\frac{1}{\omega_{s} \pm n \omega_{p}}\left(\omega_{s} \sin \varphi_{I, s} \pm n \omega_{p} \sin \varphi_{I, p}\right)(n=\ldots,-2,-1,0,+1,+2, \ldots)
$$

If $\varphi_{I, p}=\varphi_{I, s}$, we notice that $\varphi_{I, n}=\varphi_{I, s}$. Furthermore, if $\varphi_{I, p}=0$, and $\omega_{p}=2 \omega_{s}$ (degenerate parametric pumping) we obtain the peculiar result

$$
\varphi_{I,-1}=-\varphi_{I, s} \cdot\left(\varphi_{I, p}=0 ; \omega_{p}=2 \omega_{s}\right) \cdot
$$

Thus, we obtain a reflected wave, of effective frequency $\omega_{s}$, in the signal wave incidence direction.

In order to demonstrate the general nature of (1.40a) we have in figure 2 sketched the first order wave spectrum for $\varphi_{I, p}=0$, and $\omega_{p}<\omega_{s}$.

If we make use of (1.11), and (1.15) relations (1.28), and (1.29) can be written as follows, if $b_{q}$ is neglected,

$$
\alpha_{q, \pm 1}= \pm \alpha_{q, 0} \frac{\gamma}{2} \frac{\omega_{e}^{2}}{\omega_{q} \omega_{p}} \frac{1}{2 \pm \frac{\omega_{p}}{\omega_{q}}\left(1-n_{p}^{2}\right)-2 n_{p} n_{q} \cos \left(\varphi_{\mathrm{II}, p}-\varphi_{\mathrm{II}, s}\right)},\left(b_{q}=0\right)
$$

which shows that the resonance $\left(\left|\alpha_{q, \pm 1}\right| \rightarrow \infty\right)$ also depends upon the angle, $\varphi_{\mathrm{II}, p}-\varphi_{\mathrm{II}, s}$, between the wave normals of the pump and signal waves. As a matter of fact, one readily verifies from (1.41) that, independently of the form of $n_{p}$ (i.e., of the dispersion law assumed for the pump wave), $\alpha_{q, \pm 1}$ can be written in the following practical fashion

$$
\alpha_{q, \pm 1}=\alpha_{q, 0} \frac{\gamma}{2} \frac{\omega_{e}^{2}}{c_{0}^{2}} \frac{1}{\left|\bar{k}_{q \pm 1}\right|^{2}-\left|\bar{k}_{q} \pm \bar{k}_{p}\right|^{2}},\left(b_{q}=0\right)
$$

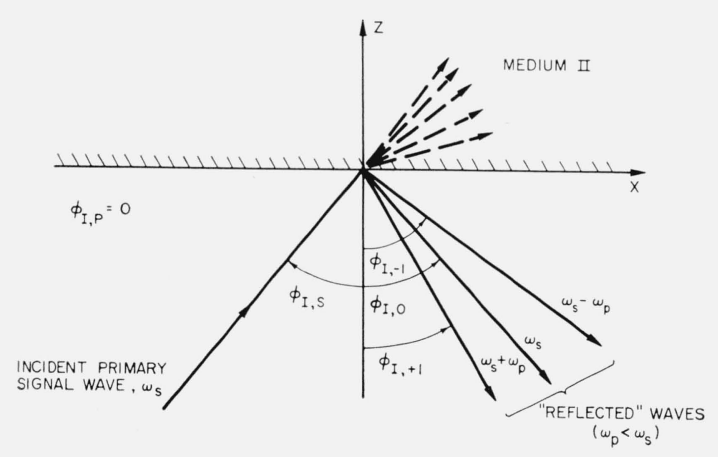

FiguRE 2. Demonstrating first order wave spectrum obtained from the pumped, ionized medium. 
which demonstrates that the system resonances are of (parametric) traveling wave type. Moreover, it is interesting to notice that the higher order coefficients (according to (1.18)) similarly can be written

and

$$
\alpha_{q, n}=\alpha_{q, n-1} \frac{\gamma}{2} \frac{\omega_{e}^{2}}{c_{0}^{2}} \frac{1}{\left|\bar{k}_{q+n}\right|^{2}-\left|\bar{k}_{q}+n \bar{k}_{p}\right|^{2}},\left(b_{q}=0\right)
$$

$$
\alpha_{q,-n}=\alpha_{q,-n+1} \frac{\gamma}{2} \frac{\omega_{e}^{2}}{c_{0}^{2}} \frac{1}{\left|\bar{k}_{q-n}\right|^{2}-\left|\bar{k}_{q}-n \bar{k}_{p}\right|^{2}},\left(b_{q}=0\right)
$$

if $\alpha_{q, n+1}$ is neglected with respect to $\alpha_{q, n-1}$, and $\alpha_{q,-n-1}$ with respect to $\alpha_{q,-n+1}$.

According to (1.42) the first order resonances appear when

and

$$
\left.\begin{array}{l}
\left|\bar{k}_{q+1}\right|= \pm\left|\bar{k}_{q}+\bar{k}_{p}\right|, \\
\left|\bar{k}_{q-1}\right|= \pm\left|\bar{k}_{q}-\bar{k}_{p}\right| .
\end{array}\right\}
$$

As will shortly be shown, it depends upon the boundary conditions which ones of these resonances that are physically possible.

If we next introduce the boundary reflection and transmission coefficients of the unperturbed signal wave, viz,

$$
R_{s, 0}=\frac{k_{0, s, z}-k_{s, z}}{k_{0, s, z}+k_{s, z}} ; T_{s, 0}=\frac{2 k_{0 . s . z}}{k_{0, s, z}+k_{s, z}},
$$

where $k_{0, s}=\omega_{s} / c_{0}$, and neglect $\alpha$-terms proportional to $\theta_{1}^{2}$ (which is not permissible in or near the instability regions), the boundary conditions $\left(E_{y}\right.$, and $H_{x}$ continuous for each frequency component) by (1.5) yield the following first order fields, if $\alpha_{0,0}$ is put equal to one.

Medium I $(z \leq 0)$

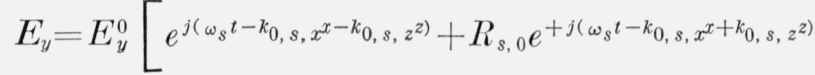

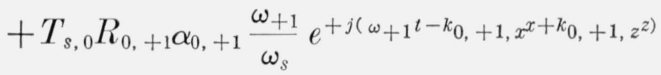

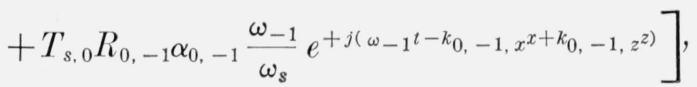

where $E_{y}^{0}$ is the amplitude of the unperturbed signal wave, and $k_{0, s, x}=k_{s, x}, k_{0, \pm 1, x}=k_{ \pm 1, x}$, which are related to each other by (1.39). The sum and difference frequency "reflection" coefficients become

$$
\begin{aligned}
& R_{0,+1}=\frac{k_{+1, z}-k_{s, z}-k_{p, z}}{k_{0,+1, z}+k_{+1, z}}\left(k_{0,+1, z}=k_{0,+1} \cos \varphi_{\mathrm{II},+1}, \text { etc. }\right) \\
& R_{0,-1}=\frac{k_{-1, z}-k_{s, z}+k_{p, z}}{k_{0,-1, z}+k_{-1, z}}\left(k_{0,-1, z}=k_{0,-1} \cos \varphi_{\mathrm{II},-1}, \text { etc. }\right)
\end{aligned}
$$

Medium II $(z \geq 0)$

$$
\begin{aligned}
& E_{y}=E_{y}^{0} T_{s, 0}\left[e^{+j\left(\omega_{s} t-k_{s, x^{x}}-k_{\left.s, z^{z}\right)}\right.}\right. \\
& +\alpha_{0,+1} \frac{\omega_{+1}}{\omega_{s}} e^{+j\left(\omega_{+1} t-k_{+1,} x\right)}\left\{e^{-j\left(k_{s, z}+k_{p, z}\right) z}-T_{0,+1} e^{-j k}+1, z^{z}\right\} \\
& \left.+\alpha_{0,-1} \frac{\omega_{-1}}{\omega_{s}} e^{+j\left(\omega_{-1} t-k\right.}-1, x^{x)}\left\{e^{-j\left(k_{s, z^{-k}}, z^{2} z\right.}-T_{0,-1} e^{-j k-1, z^{z}}\right\}\right],
\end{aligned}
$$


where the sum and difference frequency "transmission" coefficients are

$$
\begin{gathered}
T_{0,+1}=\frac{k_{0,+1, z}+k_{s, z}+k_{p, z}}{k_{0,+1, z}+k_{+1, z}}, \\
T_{0,-1}=\frac{k_{0,-1, z}+k_{s, z}-k_{p, z}}{k_{0,-1, z}+k_{-1, z}} .
\end{gathered}
$$

It is interesting to notice that, for $k_{p}=0$ (nontraveling pump wave) these reflection and transmission coefficients, $R_{0, \pm 1}, T_{0, \pm 1}$, reduce to the regular ones for a frequency shifting network.

According to (1.42)

$$
\begin{aligned}
& R_{0,+1} \alpha_{0,+1}=\frac{\gamma}{2} \frac{\omega_{e}^{2}}{c_{0}^{2}} \frac{1}{k_{0,+1, z}+k_{+1, z}} \frac{1}{k_{+1, z}+k_{s, z}+k_{p, z}}, \quad\left(\alpha_{0,0}=1\right) \\
& R_{0,-1} \alpha_{0,-1}=\frac{\gamma}{2} \frac{\omega_{e}^{2}}{c_{0}^{2}} \frac{1}{k_{0,-1, z}+k_{-1, z}} \frac{1}{k_{-1, z}+k_{s, z}-k_{p, z}}, \quad\left(\alpha_{0,0}=1\right)
\end{aligned}
$$

since $\alpha_{0, \pm 1}$ by (1.39) can be written

$$
\alpha_{0, \pm 1}=\frac{\gamma}{2} \frac{\omega_{e}^{2}}{c_{0}^{2}} \frac{1}{k_{ \pm 1, z}^{2}-\left(k_{s, z} \pm k_{p, z}\right)^{2}} \cdot \quad\left(\alpha_{0,0}=1\right) .
$$

Relation (1.55) is quite general in nature. As will be shown in section 3, for example, similar traveling wave resonance coefficients are obtained also for the much more complicated magnetoionic medium.

According to (1.53), and (1.54) only two parametric resonances are left in the system, viz,

$$
\begin{aligned}
& k_{+1, z}=-\left(k_{\mathrm{s}, z}+k_{p, z}\right) \\
& k_{-1, z}=-\left(k_{s, z}-k_{p, z}\right) .
\end{aligned}
$$

The other two resonances cancel out due to the boundary reflection effects. According to (1.50) there are two sum and two difference frequency waves generated in medium II. The terms $\exp \left\{-j\left(k_{s, z} \pm k_{p, z}\right)\right\}$ represent associated waves or "side bands" of the signal wave caused by pump modulation of the same. These waves do not in themselves satisfy the wave equation. The remaining waves, represented by the terms $T_{0, \pm 1} \exp \left\{-j k_{ \pm 1, z} z\right\}$ can be considered as natural waves ( $b_{q}$ assumed to be zero) and are, to first order, independent solutions of Maxwell's equations. Since the energy balance at the boundary $(z=0)$ of these waves, and of the sum and difference frequency waves in medium $I$, requires that the $\operatorname{sig} n$ of $k_{ \pm 1, z}$ be equal to the sign of $-\left(k_{s, z} \pm k_{p, z}\right)$, the resonance (1.56) is possible, if only the condition $\left|k_{ \pm 1, z}\right|=\left|k_{s, z} \pm k_{p, z}\right|$ can be satisfied (a situation more easily attainable in the magneto-ionic medium).

If the pump and signal waves travel in positive $z$-direction, i.e., if $k_{p, z}$ and $k_{s, z}$ are positive (which we have assumed from the beginning), the natural sum frequency wave in medium II always travels in negative $z$-direction, i.e., towards the boundary. As far as the difference frequency wave is concerned, we have to distinquish between the two cases $\omega_{p} \gtrless \omega_{s}$. If $\omega_{p}>\omega_{s}$, the natural difference frequency wave travels in positive $z$-direction if $k_{p, z}<k_{s, z}$, and in negative direction if $k_{p, 2}>k_{s, 2}$. When $\omega_{p}<\omega_{s}$, the situation is reversed.

At degenerate parametric resonance $\left|k_{-1}\right|=\left|k_{s}\right|$. This resonance therefore is possible only if $2\left|k_{s}\right| \geq k_{p}$, i.e., if $n\left(\omega_{s}\right) \geq n_{p}$. If $k_{p}$ is determined by the properties of the isotropic ionized layer only (for an unguided electromagnetic pump wave), this resonance is not possible for traveling waves.

If we assume that $k_{p}=0$ (for example if $\omega_{p}^{2}=\omega_{e}^{2}$ ), the degenerate parametric resonance requires that

$$
n_{s, z}=-j \sqrt{4+\sin ^{2} \varphi_{\mathrm{I}, 0}}=-n_{-1, z}
$$


which yields one exponentially decreasing wave (with $z$ ) in medium II, and one exponentially increasing; the energy flow associated with this pair is negative (at the frequency $\omega_{s}=\omega_{p} / 2$ ). This generates the "amplified" signal returning at the peculiar angle $-\varphi_{\mathrm{I}, s}$ from the boundary. The pumped ionized medium now acts as an "amplifying" wall, or resonant reflection amplifier, the necessary power being supplied by the pump wave. Obviously one could consider parametric plasma amplifiers operating on this principle.

\subsection{Flectron Velocity Oscillations Also Considered}

This case leads to a more involved wave equation than (1.4), since the equation of motion of the electrons now becomes

$$
\frac{\partial v_{y}}{\partial t}+v_{p, x} \frac{\partial v_{y}}{\partial x}+v_{p, z} \frac{\partial v_{y}}{\partial z}=-\frac{e}{m} E_{y}+\mu_{0} \frac{e}{m}\left(v_{p, x} H_{z}-v_{p, z} H_{x}\right) .
$$

If this relation is introduced into Maxwell's equations one obtains the following wave equation [Rydbeck, 1963],

$$
\frac{\partial}{\partial t}\left(\frac{\partial}{\partial t}+v_{p, x} \frac{\partial}{\partial x}+v_{p, z} \frac{\partial}{\partial z}\right)\left\{\frac{1}{1+\gamma \cos \left(\omega_{p} t-\bar{k}_{p} \bar{r}\right)} P_{e}^{2} \psi\right\}-\frac{\omega_{e}^{2}}{c_{0}^{2}}\left(\frac{\partial^{2}}{\partial t^{2}}+v_{p, x} \frac{\partial^{2}}{\partial t \partial x}+v_{p, z} \frac{\partial^{2}}{\partial t \partial z}\right) \psi=0
$$

where

$$
P_{e}^{2}=\frac{\partial^{2}}{\partial x^{2}}+\frac{\partial^{2}}{\partial z^{2}}-\frac{1}{c_{0}^{2}} \frac{\partial^{2}}{\partial t^{2}}
$$

Since this equation unfortunately can not be reduced to a Mathieu equation when $v_{p} \neq 0$, we solve it to first order by series expansion of the following type (quite analogous to the Mathieu series when $b_{q}=0$ ), viz,

$$
\psi_{q}=e^{+j\left(\omega_{q} t-\bar{k}_{q} \bar{r}\right)} \beta_{q}\left\{\alpha_{q, 0}+\alpha_{q,+1} e^{+j 2 u}+\alpha_{q,-1} e^{-j 2 u}+\ldots\right\} \quad\left(2 u=\omega_{p} t-\bar{k}_{p} \bar{r}\right) .
$$

Next, we put

$$
v_{p, z}=v_{p}^{0} \cos \varphi_{\mathrm{II}, p}^{(v)} \cos 2 u ; v_{p, x}=v_{p}^{0} \sin \varphi_{\mathrm{II}, p}^{(v)} \cos 2 u,
$$

where $\varphi_{I I, p}^{(v)}$ is the angle that the electron velocity of the pump wave makes with the $z$-axis. We have used $2 u$ as the general pump wave propagation factor, although this is not correct if both linear and nonlinear pump wave quantities are considered. The $\Delta N$ and the longitudinal velocity oscillations $\left(v_{L}\right)$, which constitute the nonlinear components of the high power pump wave, have a $u$-value different from that of the transverse, and linear pump wave velocity. However, for the sake of simplicity, and since it is natural to treat the effects upon the signal wave of the latter velocity separately, we use the same $u$ throughout and later adjust the velocity angle, $\varphi_{\mathrm{II}, p}^{(v)}$ and $u$ to the relevant case under study.

If we insert (1.59) and (1.60) in (1.58), and retain only the linear $\gamma$ and $v_{p}$ terms, we obtain after some rearrangement of terms, remembering that $P_{e}^{2}\left[\exp \left\{j\left(\omega_{\mathrm{q}} t-\overline{k_{\mathrm{q}}} \bar{r}\right)\right\}\right]$ $=\left(\omega_{e}^{2} / c_{0}^{2}\right) \exp \left\{j\left(\omega_{q} t-\overline{k_{q}} \bar{r}\right\}\right.$,

where

$$
a_{0, \pm 1}=\alpha_{0, \pm 1}\left\{1 \pm \frac{\tau}{\gamma} \frac{\omega_{s} \omega_{p}}{\omega_{ \pm 1}^{2}} \cos \left(\varphi_{\mathrm{II}, p}^{(v)}-\varphi_{\mathrm{II}, s}\right)\right\},\left(\text { if } a_{0,0}=1\right)
$$

$$
\tau=\frac{v_{p} n_{s}}{c_{0}}=\frac{v_{p}}{\left(v_{\text {phase }}\right)_{\omega=\omega_{s}}},
$$

which is a measure of the velocity modulation. It is important to notice from (1.61) that the system resonances, as one might expect, remain unchanged, even if the effects of the pump 
wave velocities are considered. Furthermore one notices, as is physically evident, that the electron velocities generated by the pump wave do not affect the propagation of the signal when $\left|\varphi_{\mathrm{II}}^{(v)},{ }-\varphi_{\mathrm{II}, s}\right|=\pi / 2$, i.e., if the pump velocities are transverse to the signal wave normal.

(1) Considering only the nonlinear plasma wave component of the pump wave. The pump wave in medium I now has the angular frequency $\omega_{p} / 2$. One finds [Rydbeck, 1961], that

$$
\gamma=\frac{1}{4} \frac{v_{p / 2}^{2}}{c_{0}^{2}} \frac{n_{p / 2}^{2}}{n_{p}^{2}} ; \tau=\frac{1}{4} \frac{v_{p / 2}^{2}}{c_{0}^{2}} \frac{n_{s} n_{p / 2}}{n_{p}^{2}} ; \text { and } \varphi_{\mathrm{II}, p}^{(v)}=\varphi_{\mathrm{II}, p}
$$

where $n_{p / 2}=n\left(\omega_{p} / 2\right)$, and $v_{p / 2}$ is the amplitide of the linear, i.e., the transverse pump wave velocity (of angular frequency $\omega_{p} / 2$ ).

From these relations it is evident that it is not permissible to neglect the nonlinear, longitudinal electron velocity generated by the pump wave. The effects of $\Delta N$, and $v_{p}$ are of the same magnitude, even though the system resonance is not displaced.

(2) Considering the transverse, linear pump wave velocity only. The pump wave in medium I now has the angular frequency $\omega_{p}$. By (1.61) we obtain

$$
\begin{array}{r}
a_{0, \pm 1}= \pm \frac{\omega_{e}^{2}}{c_{0}^{2}} \frac{1}{\left|\bar{k}_{ \pm 1}\right|^{2}-\left|\bar{k}_{0} \pm \bar{k}_{p}\right|^{2}} \frac{v_{p} n_{s}}{2 c_{0}} \frac{\omega_{s} \omega_{p}}{\omega_{ \pm 1}^{2}} \sin \left(\varphi_{\mathrm{II}, p}-\varphi_{\mathrm{II}, s}\right), \\
\left(a_{0,0}=1\right)
\end{array}
$$

since $\varphi_{\mathrm{II}, p}^{(v)}=\varphi_{\mathrm{II}, p}( \pm) \pi / 2$, in this case. Here we note that, for transverse pumping, i.e., the pump and signal wave crossing each other perpendicularly, the interaction will be approximately at a maximum, and much more efficient than in case 1 , where $a_{0, \pm 1}$ is proportional to $\left(v_{p} / c_{0}\right)^{2}$.

\subsection{Notes Concerning the Instabilities of the Pumped, Ionized Medium}

The instability regions, and their effects upon the wave propagation have been neglected in our previous investigations. Let us therefore, for the sake of completeness, prove that self oscillations are impossible in the infinitely extended (from $z=0$ to $+\infty$ ), ionized medium.

We now have to evaluate $b_{q}(1.25)$ for the unstable waves. It can be verified, for example from (1.43) and (1.44), that

$$
\sqrt{\theta_{0, q}} \pm n=\frac{1}{ \pm n}\left\{\left|\bar{k}_{q \pm n}\right|^{2}-\left|\bar{k}_{q} \pm n \bar{k}_{p}\right|^{2}\right\}
$$

which yields the important relations

$$
\sqrt{\theta_{0,0}} \pm n=\sqrt{\theta_{0, \pm n}} \mp n .
$$

Let us, as a typical example, study the unstable sum frequency wave. It appears from (1.28a), and (1.29a) that we get unstable $\omega_{0}$, and $\omega_{0}+\omega_{p}$ waves, if $\sqrt{\theta_{0,0}}+1 \rightarrow 0$, and if $\sqrt{\theta_{0,+1}}-1 \rightarrow 0$. We thus infer from (1.65) that these resonances are identical. Since $b_{0}$, and $b_{+1}$ by (1.25) now assume the forms,

$$
\begin{aligned}
& b_{0} \cong \sqrt{\theta_{0,0}}+1-\sqrt{\left(\sqrt{\theta_{0,0}}+1\right)^{2}-\left(-\theta_{1} / 2\right)^{2}} ;\left(\sqrt{\theta_{0,0}} \approx-1\right) \\
& b_{+1} \cong-\left\{\sqrt{\theta_{0,+1}}-1-\sqrt{\left(\sqrt{\theta_{0,+1}}-1\right)^{2}-\theta_{1}^{2} / 4}\right\} ;\left(\sqrt{\theta_{0,+1}} \approx+1\right),
\end{aligned}
$$

we notice that within the instability range in question

$$
\operatorname{Im}\left(b_{0}\right)=\operatorname{Im}\left(b_{+1}\right),\left(\sqrt{\theta_{0,0}} \cong-1 ; \sqrt{\theta_{0,+1}} \cong+1\right)
$$

which, for waves growing with time, at resonance yields

$$
b_{0}=b_{+1}=-j \theta_{1} / 2 . \quad\left(\sqrt{\theta_{0,0}}=-1 ; \sqrt{\theta_{0,+1}}=+1\right)
$$


It is now necessary to retain the $\alpha_{+1,-1}$ term in (1.32). The growing wave, of complex frequency $\omega_{0}+b_{0} \omega_{p} / 2$, by our previous relations, assuming $b_{+1}=b_{0}$, now can be written

$$
\psi^{(0)}=e^{+j\left(\omega_{0}+b_{0} \omega_{p} / 2\right) t}\left\{e^{\left.-j \bar{k}_{0}+b_{0} \bar{k}_{p} / 2\right) \bar{r}} \alpha_{0,0}+e^{-j\left(\bar{k}_{+1}-\bar{k}_{p}+b_{0} \bar{k}_{p} / 2\right) r-} \alpha_{+1,-1}\right\}, \text { (medium II) }
$$

and the corresponding sum frequency wave

$$
\psi^{(+1)}=e^{+j\left(\omega_{0}+\omega_{p}+b_{0} \omega_{p} / 2\right)} t\left\{e^{-j\left(\bar{k}_{0}+\bar{k}_{p}+b_{0} \bar{k}_{p} / 2\right) \bar{r}} \alpha_{0,+1}+e^{-j\left(\bar{k}_{+1}+b_{0} \bar{k}_{p} / 2\right) \bar{r}} \alpha_{+1,0}\right\}, \text { (medium II) }
$$

where it has to be noted that we, as before, must require that

$$
k_{+1, x}=k_{0, x}+k_{p, x} .
$$

Since we are investigating the conditions for possible self oscillations, eventual waves present in medium I must leave the interface. They therefore assume the forms

where

$$
\begin{gathered}
\xi^{(0)}=e^{+j\left(\omega_{0}+b_{0} \omega_{p} / 2\right) t+\overline{j k} 0, \overline{0^{\tau}}} d_{0},(\text { medium I) } \\
\xi^{(+1)}=e^{+j\left(\omega_{0}+\omega_{p}+b_{0} \omega_{p} / 2\right) t+\bar{j} \bar{k}_{0,+1^{\bar{r}}}} d_{+1},(\text { medium I) }
\end{gathered}
$$

$$
k_{0,0}=\frac{\omega_{0}+b_{0} \omega_{p} / 2}{c_{0}} ; \text { and } k_{0,+1}=\frac{\omega_{0}+\omega_{p}+b_{0} \omega_{p} / 2}{c_{0}} .
$$

If

$$
k_{0,0, x}=k_{0,0} \sin \varphi_{\mathrm{I}, 0} \text {, and } k_{0,+1, x}=k_{0,+1} \sin \varphi_{\mathrm{I},+1},
$$

we require that

$$
b_{0} k_{p, x} / 2=\left(b_{0} \omega_{p} / 2 c_{0}\right) \sin \varphi_{\mathrm{I}, 0}=\left(b_{0} \omega_{p} / 2 c_{0}\right) \sin \varphi_{\mathrm{I},+1},
$$

i.e., $\varphi_{\mathrm{I}, 0}=\varphi_{\mathrm{I},+1}$. Moreover,

and, by (1.70),

$$
\frac{\omega_{0}}{c_{0}} \sin \varphi_{\mathrm{I}, 0}=k_{0, x}
$$

i.e.,

$$
\frac{\omega_{0}+\omega_{p}}{c_{0}} \sin \varphi_{\mathrm{I}, 0}=k_{0, x}+k_{p, x}
$$

$$
\frac{\omega_{p}}{\omega_{0}}=\frac{k_{p . x}}{k_{0, x}}
$$

which puts an additional restriction on $\omega_{p}$ (note: $\omega_{0}$ unknown).

The boundary conditions ( $E_{y}$, and $H_{x}$ continuous for each frequency component) next yield the following two relations,

$$
\begin{gathered}
\frac{\alpha_{+1,-1}}{\alpha_{0,0}}=-\frac{k_{0,0, z}+k_{0, z}+b_{0} k_{p, z} / 2}{k_{0,0, z}+k_{+1, z}-k_{p, z}+b_{0} k_{p, z} / 2}, \\
\frac{\alpha_{+1,0}}{\alpha_{0,+1}}=-\frac{k_{0,+1, z}+k_{0, z}+k_{p, z}+b_{0} k_{p, z} / 2}{k_{0,+1, z}+k_{+1, z}+b_{0} k_{p, z} / 2} .
\end{gathered}
$$

By (1.28a), and (1.29a) we next obtain

$$
\begin{aligned}
& \frac{\alpha_{+1,0}}{\alpha_{0,0}}=-\frac{2}{\theta_{1}}\left\{\sqrt{\theta_{0,+1}}-1+\sqrt{\left(\sqrt{\theta_{0,+1}}-1\right)^{2}-\theta_{1}^{2} / 4}\right\} \cdot \frac{\alpha_{+1,-1},}{\alpha_{0,0}}, \\
& \frac{\alpha_{+1,0}}{\alpha_{0,0}}=+\frac{\theta_{1}}{2} \frac{1}{\left\{\sqrt{\theta_{0,0}}+1+\sqrt{\left(\sqrt{\theta_{0,0}}+1\right)^{2}-\left(-\theta_{1} / 2\right)^{2}}\right.} \cdot \frac{\alpha_{+1,0},}{\alpha_{0,+1}}
\end{aligned}
$$


where it is to be noted that by (1.65), (1.65a), and (1.70)

$$
\sqrt{\theta_{0,0}}+1=\sqrt{\theta_{0,+1}}-1=\left|\bar{k}_{+1}\right|^{2}-\left|\bar{k}_{0}+\bar{k}_{p}\right|^{2}=k_{+1, z}^{2}-\left(k_{0, z}+k_{p, z}\right)^{2} .
$$

Since growing, self oscillations can only be sustained, if (1.77) and (1.78) yield identical amplitude coefficient ratios $\left(\alpha_{+1,0} / \alpha_{0,0}\right)$, it is necessary that

$$
\sqrt{\theta_{0,0}}+1=0=\sqrt{\theta_{0,+1}}-1 \text {, i.e., that } k_{+1, z}= \pm\left(k_{0 . z}+k_{p, z}\right),
$$

and that

2 .

which is possible if

$$
\frac{\alpha_{+1,-1}}{\alpha_{0,0}}=\frac{\alpha_{+1,0}}{\alpha_{0,+1}}
$$

of if

$$
k_{+1, z}=k_{0, z}+k_{p, z} \text {, that yields } \frac{\alpha_{+1,-1}}{\alpha_{0,0}}=1=\frac{\alpha_{+1,0}}{\alpha_{0,+1}}
$$

$$
k_{0,+1, z}=k_{0,0, z}-k_{p, z} \text {. }
$$

Condition (1.81), which is one of the resonance conditions $(1.80)$, yields $\psi^{0}=0, \psi^{+1}=0$, and therefore also $d_{0}=0=d_{+1}$.

Condition (1.82), which yields nonvanishing fields for the resonance condition $k_{+1, z}=$ $-\left(k_{0, z}+k_{p, z}\right)$ only, by (1.73) implies that

$$
\omega_{p} \cos \varphi_{\mathrm{I}, 0} / c_{0}=-k_{p, z},
$$

which is impossible for a pump wave running in positive $z$-direction $\left(k_{p, z}>0\right)$, as has been assumed originally (pump source outside the medium).

We have thus shown that growing self oscillations are not possible for the infinitely extended (from $z=0$, to $+\infty$ ) ionized medium, which in this respect is analogous to the parametric traveling wave amplifier. This is not necessarily so for a bounded medium. A discussion of this interesting case is outside the scope of the present communication, however. And so is the case of wave reflection within the instability regions (which are very narrow for weak pumping.)

\section{Generation of Natural Sum and Difference Frequency Waves in a Slowly Inhomogeneous Medium}

We have seen in the preceding paragraph how the boundary conditions at $z=z_{0}$ determine the possible parametric traveling wave resonances and the amplitudes of the sum and difference frequency waves. Naturally one would like to know what happens in an inhomogeneous medium, if resonances occur at some specified level $z=z_{0}$.

Let us therefore consider an inhomogeneous medium, but a slowly varying one, so that partial reflections of the signal and pump waves can be neglected. Furthermore we limit ourselves, for the sake of simplicity, to an analysis of the difference frequency wave. Since the medium is slowly varying in (the $z$-direction) the signal wave assumes the form $\exp \left\{j\left(\omega_{s} t\right.\right.$ $\left.\left.-k_{s, x} x-\int^{z} k_{s, z} d z\right)\right\}$, and similarly the pump wave can be written $\cos \left(\omega_{p} t-k_{p, x} x-\boldsymbol{J}^{z} k_{p, z} d z\right)$. The natural, uncoupled difference frequency waves are written

$$
\left.\Pi^{(1)}=e^{j\left(\omega_{-1} t-k_{-1, x} x \mp \int^{z} k_{-1,2} d z\right.}\right) . \quad\left(\omega_{-1}=\omega_{s}-\omega_{p}\right) .
$$

Since $k_{-1, x}=k_{s, x}-k_{p, x}$, (1.4) now yields the following difference frequency field,

$$
\psi^{(-1)}=\alpha\left\{\Pi^{(2)} \int_{z_{1}}^{z} \Pi^{(1)} \xi d z-\Pi^{(1)} \int_{z_{3}}^{z} \Pi^{(2)} \xi d z\right\}
$$


where

and

$$
\xi=\frac{\gamma}{2} \frac{\omega_{e}^{2}}{c_{0}^{2}} e^{j\left(\omega_{-1} t-\int^{z} k_{s, z} d z+\int^{z} k_{p, z} d z\right)}
$$

$$
\alpha=e^{j 2 \omega-1 t} \frac{\Pi^{(1)}}{\Pi^{(2)}} \int^{z} \frac{d z}{\left(\Pi^{(1)}\right)^{2}} .
$$

For a slowly varying medium $\alpha \simeq \frac{1}{2 j k_{-1, z}}$, and therefore by (1.5)

$$
\begin{aligned}
& E_{y}^{(-1)} \cong E_{y}^{0} e^{j\left\{\omega-1 t-k_{-1, x}\left(x-x_{a}\right)\right\}} \frac{\omega_{-1}}{\omega_{0}} \frac{1}{2 j k_{-1, z}} \frac{\gamma}{2} \frac{\omega_{0}^{2}}{c_{0}^{2}}\left\{e^{+j \int_{z_{a}}^{z} k_{-1,2} d z} \int_{z_{1}}^{z} e^{-j \int_{z_{a}}^{z}\left(k_{s, 2}-k_{p, z}+k_{-1,2}\right) d z} d z\right. \\
& \left.-e^{-j \int_{z_{a}}^{z} k_{-1, z} d z} \int_{z_{3}}^{z} e^{-j \int_{z_{a}}^{z}\left(k_{s, z}-k_{p, z}-k_{-1, z}\right) d z} d z\right\}
\end{aligned}
$$

One notices that the two coupling integrals may "generate" natural 'difference frequency waves at or near their respective traveling wave resonance levels (compare (1.55))

and

$$
k_{-1, z}=-\left(k_{s, z}-k_{p, z}\right)
$$

$$
k_{-1, z}=+\left(k_{s, z}-k_{p, z}\right) .
$$

If, for example, $k_{p, z}<k_{s, z}$, and $\omega_{p}<\omega_{s}$, these resonances actually yield forward, natural difference frequency waves, and backward (i.e., "reflected" from the resonance region) if $k_{p, z}>k_{s, z}$.

If we assume that (2.6) holds at some specified level $z=z_{0}$, we can write as follows for the slowly varying medium

where

$$
\int_{z_{0}}^{z}\left(k_{s, z}-k_{p, z}+k_{-1, z}\right) d z=\mathcal{H}\left(z-z_{0}\right)^{2} / 2=W^{2},
$$

$$
\mathcal{H}=\left\{\frac{d}{d z}\left(k_{s, z}-k_{p, z}+k_{-1, z}\right)\right\}_{z=z_{0}}
$$

Since we only need to know the electric field of the emerging natural, difference frequency wave (i.e., the wave radiated from the interaction region), we can limit ourselves to such $z$-regions, or $\left|z-z_{0}\right|$ distances, for which $W^{2}>>1$. If we make use of the asymptotic form of the Fresnel integral, and assume that the condition for forward radiation is satisfied, relation (2.5) yields the following difference frequency fields, viz, $z<z_{0}, W^{2}>>0$

$$
E_{y}^{(-1)}=E_{y}^{0} \quad e^{j\left\{\omega_{-1} t-k_{-1, x}\left(x-x_{a}\right)-\int_{z_{u}}^{z}\left(k_{s, z}-k_{p, z}\right) d z\right\}} \frac{\omega_{-1}}{\omega_{s}} \alpha_{0,-1}, \quad\left(\alpha_{0,0}=1\right)
$$

$z>z_{0}, W^{2}>>0$

$$
\begin{aligned}
&\left.E_{y}^{(-1)}=E_{y}^{0} \quad e^{j\left\{\omega_{-1} t-k_{-1, x}\left(x-x_{a}\right)-\int_{z_{a}}^{z_{0}}\left(k_{s, z}-k_{p, z}\right) d z\right.}\right\} \frac{\omega_{-1}}{\omega_{s}}\left\{\alpha_{0,-1}\right. \\
&\left.-\frac{1}{2 k_{-1, z}} \frac{\gamma}{2} \frac{\omega_{0}^{2}}{c_{0}^{2}} \sqrt{\frac{\pi}{2 \mathcal{H}}} e^{+j\left(\int_{z_{0}}^{z} k_{-1, z} d z+\pi / 4\right)}\right\} \quad\left(\alpha_{0,0}=1\right) .
\end{aligned}
$$

Relation (2.10) is analogous to the forced difference frequency part of (1.50). This wave, (2.10), which alone does not satisfy the wave equation, is associated with the signal wave and can be considered as a "modulation" or sideband of the same. Its resonance is independent of the sign of $k_{s, z}-k_{p, z}$, as it should be. 
It appears from (2.11) that $\sqrt{\frac{2 \pi}{\mathcal{H}}}$ is a measure of the difference frequency radiation efficiency of the interaction region. If $\mathcal{H}$ is small, i.e., if the medium parameters vary very slowly, the radiation is large, provided the region is so extended that $W^{2}$ is large at its outer edges.

If we next consider the second resonance (2.7), which takes place at the same frequencies as the former one, (2.6), one finds that (2.5) yields same result as (2.10) and (2.11). The difference between the two resonances is purely formal.

If $k_{p, z} \lesseqgtr k_{s, z}$ and $\omega_{p} \gtrless \omega_{s}$, we obtain a "reflected" natural difference frequency wave, i.e., when the associated difference frequency wave (nonlinear driving force) travels in negative z-direction. When the opposite conditions hold, we get a forward difference frequency wave. Since the natural sum frequency wave radiates in the running direction of the associated sum frequency wave, a "reflected" sum frequency wave can be obtained only if the pump wave runs in negative direction and with $-k_{p, z}>k_{s, z}$. This is in contrast to the case of the disboundary, which always yields also "reflected" sum and difference frequency waves.

The amplitude of the difference frequency reflection coefficient now can be written

$$
\left|R_{0,-1}\right|=\frac{\omega_{-1}}{\omega_{s}} \frac{\gamma}{2} \frac{\omega_{e}^{2}}{c_{0}^{2}} \frac{1}{2\left|k_{-1, z}\right|} \sqrt{\frac{2 \pi}{|\mathcal{H}|}},
$$

which corresponds to

$$
\left|T_{s, 0} R_{0,-1} \alpha_{0,-1} \frac{\omega_{-1}}{\omega_{s}}\right| \text { of }(1.47) .
$$

The frequency dependent phase of the frequency shifted "echo" is

$$
\phi_{0,-1}=\int_{z_{a}}^{z_{0}} \underbrace{\left(k_{s, z}-k_{p, z}-k_{-1, z}\right) d z}_{\approx 2\left(k_{s, z}-k_{p, z}\right)} \quad\left(\omega_{p}>\omega_{s}\right) .
$$

Since $z_{0}$ depends upon $\omega_{s}$ (as well as $\omega_{p}$ ), the echo delay, $\tau$, counted at the level $z_{a}$ for a pulsed signal wave $\left(\varphi_{\mathrm{I}, s}\right.$ and $\varphi_{\mathrm{I}, p}$ assumed to be zero) becomes

$$
\tau=\frac{\partial \phi_{0,-1}}{\partial \omega_{s}} \simeq\left\{z_{0}-z_{a}+\left(\frac{2 k_{-1, z}}{\mathcal{H}}\right)_{z_{0}}\right\}\left(\frac{1}{v_{g, s}}+\frac{1}{v_{g,-1}}\right)
$$

where $v_{g, s}$ and $v_{g,-1}$ are the group velocities of the signal wave and the natural difference frequency wave. For a forward wave emerging at $z=z_{b}\left(>z_{0}\right)$, we similarly obtain

$$
\tau \cong\left(z_{0}-z_{a}\right) \frac{1}{v_{g, s}}+\left(z_{b}-z_{0}\right) \frac{1}{v_{g,-1}}+\left(\frac{2 k_{-1, z}}{\mathcal{H}}\right)_{z_{0}}\left(\frac{1}{v_{g, s}}-\frac{1}{v_{g,-1}}\right) .
$$

We thus get a change in effective reflection height of $\left(k_{p, z} / \mathcal{H}()_{z o}\right.$ which may be a large quantity. It is a measure of the "frequency shift reflection thickness" of the interaction region. As the dispersion is very large, it is not sufficient to make use of the first phasefrequency derivative only to determine the time of arrival of the frequency shifted wave. Such a discussion lies outside the scope of the present communication.

Since

$$
\left(k_{-1, z}\right)_{z 0}=-\left(k_{s, z}-k_{p, z}\right)_{z 0}
$$

and

$$
k_{-1, x}=k_{s, x}-k_{p, x}
$$

one can sketch the frequency shifted wave normal paths about as shown in figure 3 . 
FIGURE 3. Demonstrating wave normal path of the frequency shifted downcoming wave.

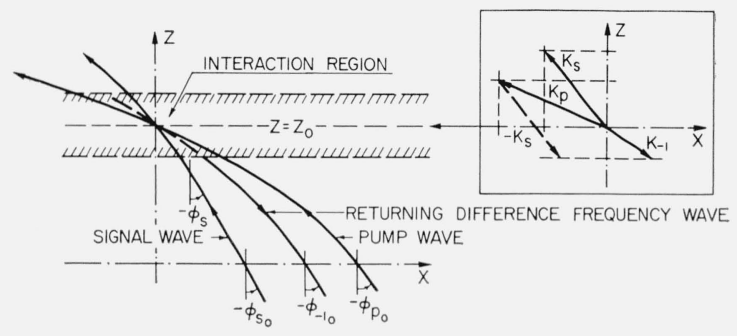

\section{Electromagnetic Wave Reflection From the Oscillating Magneto-Ionic Medium}

Let us assume that the static magnetic field lies in the $x-z$ plane, and that its electronic angular gyrofrequency is $\omega_{H}$, with components $\omega_{L}=\omega_{H} \cos \theta, \omega_{T}=\omega_{H} \sin \theta$, along the $\approx$ and $x$ axes, respectively.

If we introduce the following operators, viz,

$$
P_{e}^{2}=-\frac{1}{\mu_{0} \epsilon_{0}} \frac{\partial^{2}}{\partial z^{2}}+\frac{\partial^{2}}{\partial t^{2}} ; P_{e m}^{2}=P_{e}^{2}+\omega_{e}^{2} ; \text { and } P_{p}^{2}=\frac{\partial^{2}}{\partial t^{2}}+\omega_{e}^{2},
$$

and limit ourselves to the case $\varphi_{\mathrm{I}, s}=0=\varphi_{\mathrm{I}, p}$ (i.e., vertical incidence) we obtain the following nonlinear wave equations for the (second order) electron velocities, $v_{y}^{(2)}, v_{x}^{(2)}, v_{z}^{(2)}$, [from the nonlinear magneto-ionic theory, Rydbeck, 1962].

$$
\begin{gathered}
D v_{y}^{(2)}=P_{p}^{2}\left(\omega_{L} P_{e}^{2} \psi_{x}+P_{e m}^{2} \frac{\partial \psi_{y}}{\partial t}\right)-\omega_{T} P_{e m}^{2} P_{e}^{2} \psi_{z}=\Pi_{y}, \\
D v_{x}^{(2)}=P_{p}^{2}\left(\omega_{L} P_{e}^{2} S_{1}^{(2)} \psi_{x}+P_{e m}^{2} S_{2}^{(2)} \frac{\partial \psi_{y}}{\partial t}\right)-\omega_{T} P_{e m}^{2} P_{e}^{2} S_{2}^{(2)} \psi_{z}=\Pi_{x}, \\
D \frac{\partial v_{z}^{(2)}}{\partial t}=\omega_{T}\left(\omega_{L} P_{e}^{2} \frac{\partial^{2} \psi_{x}}{\partial t^{2}}+P_{e m}^{2} \frac{\partial^{3} \psi_{y}}{\partial t^{3}}\right)+\underbrace{\left(P_{e m}^{4} \frac{\partial^{2}}{\partial t^{2}}+\omega_{L}^{2} P_{e}^{4}\right)}_{a} \psi_{z}=\Pi_{2},
\end{gathered}
$$

where $\Pi_{y}, \Pi_{x}, \Pi_{z}$ are nonlinear driving terms to be specified later. It is worth noting that $a$ annihilates longitudinal magneto-ionic modes.

Furthermore

$$
D=P_{e m}^{2}\left(P_{e m}^{2} P_{p}^{2}+\omega_{T}^{2} P_{e}^{2}\right) \frac{\partial^{2}}{\partial t^{2}}+\omega_{L}^{2} P_{p}^{2} P_{e}^{4}
$$

By (3.5) relation (3.4) can be given the alternate form

$$
P_{p}^{2} \frac{\partial v_{z}^{(2)}}{\partial t}=\omega_{T} \frac{\partial^{2} v_{y}^{(2)}}{\partial t^{2}}+\psi_{z}
$$

The second order polarizations, $S_{1}^{(2)}$ and $S_{2}^{(2)}$, are related to each other as follows

$$
S_{1}^{(2)}=\frac{\left(P_{p}^{2} P_{e m}^{2}+\omega_{T}^{2} P_{e}^{2}\right) \partial / \partial t}{\omega_{L} P_{p}^{2} P_{e}^{2}} ; S_{2}^{(2)}=-\frac{\omega_{L} P_{e}^{2}}{P_{e m}^{2} \partial / \partial t} ; S_{1}^{(2)}=S_{2}^{(2)}\left(1-\frac{D}{\omega_{L}^{2} P_{p}^{2} P_{e}^{4}}\right)
$$

Relation (3.3) can now be transformed to

$$
v_{x}^{(2)}=S_{2}^{(2)} v_{y}^{(2)}+\frac{\psi_{x}}{P_{e m}^{2} \partial / \partial t}=S_{2}^{(2)}\left(v_{y}^{(2)}-\frac{\psi_{x}}{\omega_{L} P_{e}^{2}}\right) .
$$


Thus $S_{1}^{(2)}=S_{2}^{(2)}$, when $D=0$, i.e., when one has system (traveling wave) resonance. When this is the case $v_{x}^{(2)}=S_{2}^{(2)} v_{y}^{(2)}$.

$D$ can be written

$$
\begin{aligned}
& P_{p}^{2} D=\underbrace{P_{p}^{2}\left\{\left[P_{e}^{2}\left(1+\frac{\omega_{T}^{2} / 2}{P_{p}^{2}}\right)+\omega_{e}^{2}\right] \frac{\partial}{\partial t}-P_{e}^{2} \sqrt{\left(\frac{\omega_{T}^{2} / 2}{P_{p}^{2}}\right)^{2} \frac{\partial^{2}}{\partial t^{2}}-\omega_{L}^{2}}\right\}}_{D_{0}} \\
& \times \underbrace{P_{p}^{2}\left\{\left[P_{e}^{2}\left(1+\frac{\omega_{T}^{2} / 2}{P_{p}^{2}}\right)+\omega_{e}^{2}\right] \frac{\partial}{\partial t}+P_{e}^{2} \sqrt{\left(\frac{\omega_{T}^{2} / 2}{P_{p}^{2}}\right)^{2} \frac{\partial^{2}}{\partial t^{2}}-\omega_{L}^{2}}\right\}}_{D_{x}} .
\end{aligned}
$$

$L_{0}$ annihilates the natural ordinary magneto-ionic mode and the plasma oscillation (for which $P_{p}^{2}=0$ ), whereas $D_{x}$ annihilates the natural extraordinary mode only. $D$ can be used to study the transient behavior of the magneto-ionic medium.

By (3.9) one can also write

$$
D=\underbrace{\left\{\left(\omega^{2}-\omega_{e}^{2}\right)\left(\omega^{2}-\omega_{H}^{2}\right)-\omega_{e}^{2} \omega_{T}^{2}\right\}}_{b} c_{0}^{4}\left\{\frac{\partial^{2}}{\partial z^{2}}+k_{0}^{2}(\omega)\right\}\left\{\frac{\partial^{2}}{\partial z^{2}}+k_{x}^{2}(\omega)\right\},
$$

where

$$
k_{0}(\omega)=\frac{\omega}{c_{0}} n_{0}(\omega) ; \text { and } k_{x}(\omega)=\frac{\omega}{c_{0}} n_{x}(\omega)
$$

and $n_{0}$ and $n_{x}$ denote ordinary and extraordinary refractive indices respectively. One notices that $b=0$ at the fourth reflection level (referred to the angular frequency) at which $\left|n_{x}\right|^{2}=\infty$. In the neighborhood of this level $D$ is conveniently written

$$
D=-P_{e}^{4} P_{p}^{2} X_{d} \omega_{e}^{2}\left(\frac{1}{1-n_{0}^{2}}-\frac{1}{1-r_{d}^{2}}\right)\left(\frac{1}{1-n_{x}^{2}}-\frac{1}{1-n_{d}^{2}}\right), \quad\left(X_{d}=\omega_{e}^{2} / \omega_{d}^{2}\right),
$$

where $n_{d}$ is the effective refractive index of the wave, of angular frequency $\omega_{d}$, upon which $D$ operates.

Next, let us introduce the driving force polarization ratio

$$
S_{d}=j Q_{d}=j \frac{\omega_{L}}{\omega_{d}} \frac{1-n_{d}^{2}}{1-X_{d}-n_{d}^{2}} .
$$

Relation (3.2) now yields

$$
\Pi_{y}=\omega_{d}^{5}\left(1-X_{d}\right)\left(1-X_{d}-n_{d}^{2}\right)\left\{Q_{a} \psi_{x}+j \psi_{y}+Y_{T, d}\left(1-n_{d}^{2}\right) \psi_{z}\right\} \quad\left(Y_{T, d}=\omega_{T} / \omega_{d}\right) .
$$

Ii the pump (high power) wave has the $v_{y}$ velocity

$$
v_{p, y}=v_{p} \cos \left(\omega_{p} t-k_{p} z\right), \quad\left(k_{p}=\omega_{p} n_{p} / c_{0}\right)
$$

where, quite arbitrarily, $k_{p}$ could refer to an ordinary or extraordinary pump wave, the other pump wave velocities become

$$
v_{p, x}=-Q_{p} v_{p} \sin \left(\omega_{p} t-k_{p} z\right) ; v_{p, z}=v_{p} \frac{Y_{T, p}}{1-X_{p}} \sin \left(\omega_{p} t-k_{p} z\right), \quad\left(X_{p}=\omega_{e}^{2} / \omega_{p}^{2}\right)
$$

with

$$
Q_{p}=Y_{L, p} \frac{1-n_{p}^{2}}{1-X_{p}-n_{p}^{2}} \cdot \quad\left(Y_{L, p}=\omega_{L} / \omega_{p}\right)
$$


The differential space charge density becomes

$$
\rho_{p}=\rho_{0} \frac{n_{p}}{c_{0}} \frac{Y_{T, p}}{1-X_{p}} v_{p} \sin \left(\omega_{p} t-k_{p} z\right) . \quad\left\{\begin{array}{l}
\rho_{0}=-e N_{e} \\
Y_{T, p}=\omega_{T /}^{\prime} \omega_{p}
\end{array}\right\} .
$$

The signal wave, of angular frequency $\omega_{s}$, is written

$$
\begin{aligned}
& v_{s, y}=v_{s} e^{j\left(\omega_{s} t-k_{s} z\right)} ; v_{s, x}=-Q_{s} \frac{v_{s}}{j} e^{j\left(\omega_{s} t-k_{s} z\right)} ; v_{s, z}=\frac{Y_{T, s}}{1-X_{s}} \frac{v_{s}}{j} e^{j\left(\omega_{s} t-k_{s} z\right)} ; \\
& \rho_{s}=\rho_{0} \frac{n_{s}}{c_{0}} \frac{Y_{T, s}}{1-X_{s}} \frac{v_{s}}{j} e^{j\left(\omega_{s} t-k_{s} z\right)}, \quad\left(k_{s}=\omega_{s} n_{s} / c_{0} ; X_{s}=\omega_{e}^{2} / \omega_{s}^{2} ; Y_{T, s}=\omega_{T} / \omega_{s}\right)
\end{aligned}
$$

where

$$
Q_{s}=Y_{L, s} \frac{1-n_{s}^{2}}{1-X_{s}-n_{s}^{2}} . \quad\left(Y_{L, s}=\omega_{L} / \omega_{s} ; Y_{T, s}=\omega_{T} / \omega_{s}\right) .
$$

$\psi_{x}, \psi_{y}, \psi_{z}$ can be written [Rydbeck, 1962]

$$
\psi_{x}=P_{e}^{2} P_{x}-\omega_{e}^{2} \frac{\partial W_{x}}{\partial t} ; \psi_{y}=P_{e}^{2} P_{y}-\omega_{e}^{2} \frac{\partial W_{y}}{\partial t} ; \psi_{z}=\frac{\partial^{2}}{\partial t^{2}} P_{z}-\omega_{e}^{2} \frac{\partial W_{z}}{\partial t} .
$$

If we limit ourselves to a study of the sum and difference frequency terms only, we find that

and

$$
\begin{gathered}
P_{x}=P_{x_{s d}}=-v_{p, z} \frac{\partial v_{s, x}}{\partial z}\left(1-\frac{X_{s}}{1-n_{s}^{2}}\right)-v_{s, z} \frac{\partial v_{p, x}}{\partial z}\left(1-\frac{X_{p}}{1-n_{p}^{2}}\right) \\
P_{y}=P_{y_{s} d}=-v_{p, z} \frac{\partial v_{s, y}}{\partial z}\left(1-\frac{X_{s}}{1-n_{s}^{2}}\right)-v_{s, z} \frac{\partial v_{p, y}}{\partial z}\left(1-\frac{X_{p}}{1-n_{p}^{2}}\right), \\
P_{z}=P_{z_{s l}}=-\frac{\partial}{\partial z}\left(v_{s, z} v_{p, z}\right)-\frac{X_{s}}{1-n_{s}^{2}}\left(\frac{\partial v_{s, y}}{\partial z} v_{p, y}+\frac{\partial v_{s, x}}{\partial z} v_{p, x}\right) \\
-\frac{X_{p}}{1-n_{p}^{2}}\left(\frac{\partial v_{p, y}}{\partial z} v_{s, y}+\frac{\partial v_{p, x}}{\partial z} v_{s, x}\right),
\end{gathered}
$$

We evaluate next $P_{x}, P_{y}, P_{z}$, and $\bar{W}$ from these relations and obtain for the sum and difference terms (index + and - , respectively)

$$
\begin{aligned}
& \psi_{x}^{ \pm}=\mp j \frac{v_{s} v_{p}}{2} \omega_{ \pm}^{2}\left[\left(1-n_{ \pm}^{2}\right)\left\{\alpha_{s} k_{p} Q_{p}\left(1-\frac{X_{p}}{1-n_{p}^{2}}\right)+\alpha_{p} k_{s} Q_{s}\left(1-\frac{X_{s}}{1-n_{s}^{2}}\right)\right\}\right. \\
& \left.+X_{ \pm} \frac{\omega_{ \pm}}{c_{0}}\left(\alpha_{s} n_{s} Q_{p}+\alpha_{p} n_{p} Q_{s}\right)\right] e^{j\left(\omega_{ \pm}{ }^{t-k_{ \pm}}{ }^{z)}\right.},^{2} \\
& \psi_{y}^{ \pm}=-\frac{v_{s} v_{p}}{2} \omega_{ \pm}^{2}\left[\left(1-n_{ \pm}^{2}\right)\left\{\alpha_{s} k_{p}\left(1-\frac{X_{p}}{1-n_{p}^{2}}\right) \pm \alpha_{p} k_{s}\left(1-\frac{X_{s}}{1-n_{s}^{2}}\right)\right\}+X_{ \pm} \frac{\omega_{ \pm}}{c_{0}}\left(\alpha_{s} n_{s} \pm \alpha_{p} n_{p}\right)\right] e^{j\left(\omega_{ \pm}{ }^{\left.t-k_{ \pm}{ }^{2}\right)},\right.} \\
& \psi_{z}^{ \pm}=j \frac{v_{s} v_{p}}{2} \omega_{ \pm}^{2}\left[ \pm \alpha_{s} \alpha_{p}\left\{k_{ \pm}+X_{ \pm} \frac{\omega_{ \pm}}{c_{0}}\left(n_{s}+n_{p}\right)\right\}-\left(\frac{X_{s}}{1-n_{s}^{2}}+\frac{X_{p}}{1-n_{p}^{2}}\right)\left(1 \mp Q_{s} Q_{p}\right)\right] e^{j\left(\omega_{ \pm}{ }^{\left.t-k_{ \pm}{ }^{2}\right)}\right.},
\end{aligned}
$$

where

$$
k_{ \pm}=k_{s} \pm k_{p} ; X_{ \pm}=\omega_{e}^{2} / \omega_{ \pm}^{2} ; \alpha_{s}=Y_{T, s} /\left(1-X_{s}\right) ; \text { and } \alpha_{p}=Y_{T, p} /\left(1-X_{p}\right) .
$$


We now write (3.14) as follows:

$$
\Pi_{y}^{ \pm}=\omega_{ \pm}^{5}\left(1-X_{ \pm}\right)\left(1-X_{ \pm}-n_{ \pm}^{2}\right)\{\underbrace{Q_{ \pm} \psi_{x}^{ \pm}+j \psi_{\nu}^{ \pm}+Y_{T, \pm}\left(1-n_{ \pm}^{2}\right) \psi_{z}^{ \pm}}_{-j \frac{v_{s} v_{p}}{2} \xi^{ \pm}}\},
$$

where

$$
Q_{ \pm}=Q_{d}\left(\omega_{d}=\omega_{ \pm}\right) ; Y_{T, \pm}=\omega_{T} / \omega_{ \pm}
$$

and by (3.26), (3.27), and (3.28) obtain

$$
\begin{aligned}
& \xi^{ \pm}=\omega_{ \pm}^{2}\left[( 1 - n _ { \pm } ^ { 2 } ) \left\{\alpha_{s} k_{p}\left(1-\frac{X_{p}}{1-n_{p}^{2}}\right)\left(1 \pm Q_{ \pm} Q_{p}\right)\right.\right. \\
& \pm \alpha_{p} k_{s}\left(1-\frac{X_{s}}{1-n_{s}^{2}}\right)\left(1 \pm Q_{ \pm} Q_{s}\right)+Y_{T, \pm}\left[ \pm \alpha_{s} \alpha_{p}\left\{k_{ \pm}+X_{ \pm} \frac{\omega_{ \pm}}{c_{0}}\left(n_{s}+n_{p}\right)\right\}\right. \\
& \left.\left.-\left(\frac{X_{s} k_{s}}{1-n_{s}^{2}}+\frac{X_{p} k_{p}}{1-n_{p}^{2}}\right)\left(1 \mp Q_{s} Q_{p}\right)\right]\right\}+X_{ \pm} \frac{\omega_{ \pm}}{c_{0}}\left\{\alpha_{s} n_{s}\left(1 \pm Q_{ \pm} Q_{p}\right)\right. \\
& \left.\left. \pm \alpha_{p} n_{p}\left(1+Q_{ \pm} Q_{p}\right)\right\}\right] e^{j\left(\omega_{ \pm}{ }^{\left.t-k_{ \pm} z\right)}\right.} \text {. }
\end{aligned}
$$

First we notice that $\xi^{ \pm}=0$, if $\omega_{T}=0$. This is due to the fact that the differential space charges and the longitudinal velocities (which form longitudinal plasma wave pairs coupled to the transverse wave by $\omega_{T}$ ) are zero in this case. Had we considered second order terms in the pump wave field (which could easily have been done) this would not have been so. The transverse magnetic field thus is a very important factor in the nonlinear interaction theory.

When $X_{s} \rightarrow 1$, or $X_{p} \rightarrow 1$, we have longitudinal driving force plasma resonance and $\left|\xi^{ \pm}\right| \rightarrow \infty$ (in the collisionless case). In this connection it is worth noting that

$$
\left.\begin{array}{l}
\frac{X}{1-n_{x}^{2}} \rightarrow-\frac{Y_{T}^{2}}{1-X}+1-\frac{Y_{L}^{2}}{Y_{T}^{2}}(1-X) ; \\
\frac{X}{1-n_{0}^{2}} \rightarrow 1+\frac{Y_{L}^{2}}{Y_{T}^{2}}(1-X),
\end{array}\right\} \text { if } X \rightarrow 1\left(Y_{T}^{2}>>\left|2(1-X) Y_{L}\right|\right)
$$

If these driving force resonances occur in the traveling wave resonance region, the generation of sum and difference frequency wave may be greatly enhanced. Even if there is no resonance of the latter kind, sudden driving force resonances may cause appreciable sum and difference frequency radiation.

Another level of considerable interest is the fourth reflection one, where $\left|n_{s}\right|$ or $\left|n_{p}\right| \rightarrow \infty$. At this level, determined by $b=0$ (which indicates that it is a gyroplasma oscillation resonance coupling), all electron velocities (and the differential space charge density) become very large. This is in contrast to the plasma resonance level, where only the longitudinal velocity becomes large (and $\rho_{s / p}$, if $n_{s / p}=n_{x}$ ).

In ionospheric applications this important resonance level is difficult to reach, except from a topside sounder. Moreover, traveling wave resonances, which are necessary, since $D$ otherwise would grow to large values [see (3.10)], might be critical to obtain at this level in practice ( $b$ would have to be zero both for $\omega_{ \pm}$and for $\omega_{s}$ or $\omega_{p}$ ).

Before we continue the discussion, let us express the driving force velocities in the corresponding $E_{\nu}$ fields. We have

$$
v_{s}=\frac{1-n_{s}^{2}}{X_{s}} \frac{e}{m \omega_{s}} E_{s, y}^{0}, \text { and } v_{p}=\frac{1-n_{p}^{2}}{X_{p}} \frac{e}{m \omega_{p}} E_{p, y}^{0},
$$


and find that

$$
\begin{gathered}
E_{y}^{ \pm}=-j \frac{m \omega_{ \pm}}{e} \frac{X_{ \pm}}{1-n_{ \pm}^{2}}\left\{-j \frac{v_{s} v_{p}}{2}\left(n_{s} \alpha_{s} \pm n_{p} \alpha_{p}\right) \frac{1}{c_{0}} e^{j\left(\omega_{ \pm}{ }^{t-k_{ \pm}} z\right)}+v_{\nu}^{(2)}\right\} \\
E_{x}^{ \pm}=-j \frac{m \omega_{ \pm}}{e} \frac{X_{ \pm}}{1-n_{ \pm}^{2}}\left\{ \pm \frac{v_{s} v_{p}}{2}\left(n_{s} \alpha_{s} Q_{p}+n_{p} \alpha_{p} Q_{s}\right) \frac{1}{c_{0}} e^{j\left(\omega_{ \pm} t-k_{ \pm} z\right)}+v_{x}^{(2)}\right\}
\end{gathered}
$$

We now obtain the final formulae,

$$
E_{v}^{ \pm}=-\frac{E_{s, y}^{0} E_{p, y}^{0}}{2} \frac{\tau_{ \pm}}{\tau_{s} \tau_{p}}\left\{\omega_{ \pm}^{5}\left(1-X_{ \pm}\right)\left(1-X_{ \pm}-n_{ \pm}^{2}\right) \xi^{ \pm} / D^{ \pm}+\frac{n_{s} \alpha_{s} \pm n_{p} \alpha_{p}}{c_{0}} e^{j\left(\omega_{ \pm}{ }^{t-k_{ \pm}} z\right)}\right\}
$$

and

$$
\begin{aligned}
E_{x}^{ \pm}=-\frac{E_{s, y}^{0} E_{p, y}^{0}}{2} \frac{\tau_{ \pm}}{\tau_{s} \tau_{p}}\left\{j Q_{ \pm} \omega_{ \pm}^{5}\left(1-X_{ \pm}\right)\left(1-X_{ \pm}-n_{ \pm}^{2}\right) \xi^{ \pm} / D^{ \pm}\right. & \\
& \left. \pm j \frac{n_{t_{s}} \alpha_{s} Q_{p}+n_{p} \alpha_{p} Q_{p}}{c_{0}} e^{j\left(\omega_{ \pm} t-k_{ \pm} z\right)}\right\}+\tau_{ \pm} \frac{\psi_{x}}{\omega_{ \pm}^{3}\left(1-X_{ \pm}-n_{ \pm}^{2}\right)},
\end{aligned}
$$

where

$$
\tau_{p}=\frac{X_{p}}{1-n_{p}^{2}} \frac{m \omega_{p}}{e}, \text { etc. }
$$

In order to study the stationary solutions, we present $D(3.10)$ in an alternate form, viz,

where

$$
D^{ \pm}=X_{ \pm}^{2}\left(1-X_{ \pm}\right) \frac{\left(n_{ \pm}^{2}-n_{0, \pm}^{2}\right)\left(n_{ \pm}^{2}-n_{x, \pm}^{2}\right)}{\left(1-n_{0, \pm}^{2}\right)\left(1-n_{x, \pm}^{2}\right)}
$$

$$
n_{ \pm}=k_{ \pm} / \omega_{ \pm} ; n_{0, \pm}=n_{0}\left(\omega_{ \pm}\right) ; \text {and } n_{x, \pm}=n_{x}\left(\omega_{ \pm}\right)
$$

Since we want to study what happens at or near the traveling wave resonances $\left(D^{ \pm}=0\right)$, especially to obtain the amplitudes of the natural sum and difference frequency waves, we neglect the (in this particular case) insignificant last terms in (3.36) and (3.37). By (3.38) we can write

$$
\begin{gathered}
E_{y}^{ \pm}=-\frac{E_{s, y}^{0} E_{p, y}^{0}}{2} \frac{e}{m \omega_{e}} \frac{\omega_{p} \omega_{s}}{\omega_{e}^{5}} \frac{\left(1-n_{p}^{2}\right)\left(1-n_{s}^{2}\right)}{1-n_{ \pm}^{2}}\left(1-X_{ \pm}-n_{ \pm}^{2}\right) \cdot \frac{\left(1-n_{0, \pm}^{2}\right)\left(1-n_{x, \pm}^{2}\right)}{\left(n_{ \pm \pm}^{2}-n_{0, \pm}^{2}\right)\left(n_{ \pm}^{2}-n_{x, \pm}^{2}\right)} \cdot \xi^{ \pm} \\
E_{x}^{ \pm}=j Q^{ \pm} E_{\nu}^{ \pm}
\end{gathered}
$$

Next, let us, in analogy with paragraph 1, determine the natural waves, that leave, or approach, the boundary $z=0$ between the ionized medium (medium II) and vacuum (medium I).

We have the following sum and difference frequency waves to consider:

\section{Medium II}

$$
\left.\begin{array}{l}
E_{x}=E_{x}^{ \pm}+T_{0, \pm 1}^{(0)} e^{j\left(\omega_{ \pm} t-k_{0, \pm} z\right)}+T_{0, \pm 1}^{(x)} e^{j\left(\omega_{ \pm} t-k_{x, \pm} z\right)} \\
\left.E_{y}=E_{y}^{ \pm}+\frac{1}{j Q_{0, \pm}} T_{0, \pm 1}^{(0)} e^{j\left(\omega_{ \pm} t-k_{0, \pm} z\right)}+\frac{1}{j Q_{x, \pm}} T_{0, \pm 1}^{(x)} e^{j\left(\omega_{ \pm} t-k x, \pm\right.} z\right)
\end{array}\right\}
$$

where $j Q_{0, \pm}$ and $j Q_{x, \pm}$ are sum and difference frequency polarization ratios of the natural sum and difference frequency waves.

\section{Medium I}

$$
\left.\begin{array}{l}
E_{x}=R_{0, \pm 1}^{(1)} e^{j\left(\omega_{ \pm} t+\omega_{ \pm} z / c_{0}\right)} \\
E_{y}=R_{0, \pm 1}^{(2)} e^{j\left(\omega_{ \pm} t+\omega_{ \pm} z / c_{0}\right)}
\end{array}\right\}
$$


If we break up the associated wave, $E_{y}^{ \pm}$, in two components, $\alpha_{ \pm}$and $\beta_{ \pm}$, with the (natural) polarization ratios $Q_{0, \pm}$ and $Q_{x, \pm}$, we obtain

$$
\left.\begin{array}{l}
\alpha_{ \pm}=-\frac{Q_{ \pm} / Q_{x, \pm}-1}{Q_{0, \pm}^{2}+1} \\
\beta_{ \pm}=-Q_{0, \pm}^{2} \frac{Q_{ \pm} / Q_{0, \pm}-1}{Q_{0, \pm}^{2}+1}
\end{array}\right\}
$$

The boundary conditions now yield

$$
\begin{aligned}
& T_{0, \pm 1}^{(0)}=-\alpha_{ \pm} j Q_{0, \pm} \frac{1-n_{ \pm}}{1-n_{0, \pm}} ; T_{0, \pm 1}^{(x)}=-\beta_{ \pm} j Q_{x, \pm} \frac{1-n_{ \pm}}{1-n_{x, \pm}}, \\
& \left.\begin{array}{l}
R_{0, \pm 1}^{(1)}=\alpha_{ \pm} j Q_{0, \pm} \frac{n_{ \pm}-n_{0, \pm}}{1-n_{0, \pm}}+\beta_{ \pm} j Q_{x, \pm} \frac{n_{ \pm}-n_{x, \pm}}{1-n_{x, \pm}} \\
R_{0, \pm 1}^{(2)}=\alpha_{ \pm} \frac{n_{ \pm}-n_{0, \pm}}{1-n_{0, \pm}}+\beta_{ \pm} \frac{n_{ \pm \pm}-n_{x, \pm}}{1-n_{x, \pm}}
\end{array}\right\} .
\end{aligned}
$$

The "reflected" field, i.e., the wave leaving the boundary $(z=0)$ in medium I, thus is composed of two parts, with polarizations $Q_{0, \pm}$ and $Q_{x, \pm}$, respectively. Since the energy flow in the $z$-direction is

$$
P_{q}^{(z)}=n_{q}\left(Q_{q}^{2}+1\right)\left|E_{y}^{0}\right|^{2} / Z_{0},
$$

the energy balance at the boundary requires that

$$
\left.\begin{array}{l}
\operatorname{Sign}\left(n_{0, \pm}\right)= \\
\operatorname{Sign}\left(n_{x, \pm}\right)=
\end{array}\right\}-\operatorname{Sign} \frac{\omega_{s} n_{s} \pm \omega_{p} n_{p}}{\omega_{s} \pm \omega_{p}} .
$$

For sum frequency waves $n_{0, \pm}$ and $n_{x, \pm}$ are thus always negative (if we assume $k_{p}$ to be positive, i.e., a pump wave incident upon the boundary in medium I). The natural sum frequency modes travel always toward the boundary in medium II. When $\omega_{p}<\omega_{s}$, the natural difference frequency modes travel towards the boundary, if $k_{p}\left\langle k_{s}\right.$, and away from it, when $k_{p}>k_{s}$. When $\omega_{p}>$ $\omega_{s}$ the situation is reversed.

If we make use of the fact that

and

$$
\frac{Q_{ \pm}}{Q_{0, \pm}}-1=\frac{X_{ \pm}}{1-X_{ \pm}-n_{ \pm}^{2}} \frac{n_{ \pm}^{2}-n_{x, \pm}^{2}}{1-n_{x, \pm}^{2}}
$$

$$
\frac{Q_{ \pm}}{Q_{x, \pm}}-1=\frac{X_{ \pm}}{1-X_{ \pm}-n_{ \pm}^{2}} \frac{n_{ \pm}^{2}-n_{0, \pm}^{2}}{1-n_{x, \pm}^{2}}
$$

the "reflected," frequency shifted field in medium I can finally be written

$$
\begin{aligned}
& E_{y}=\frac{E_{s, y}^{0} E_{p, y}^{0}}{2} \frac{e}{m \omega_{ \pm}} \frac{\omega_{p} \omega_{s}}{\omega_{e}^{4} \omega_{ \pm}} \frac{\left(1-n_{p}^{2}\right)\left(1-n_{s}^{2}\right)}{1-n_{ \pm}^{2}}\left\{\frac{1}{Q_{0, \pm}^{2}+1} \frac{1+n_{0, \pm}}{n_{ \pm}+n_{0, \pm}}+\frac{1}{Q_{x, \pm}^{2}+1} \frac{1+n_{x, \pm}}{n_{ \pm}+n_{0, \pm}}\right\} \xi^{ \pm} e^{j \omega_{ \pm} z / c_{0}}, \\
& E_{x}=\frac{E_{s, y}^{0} E_{p, y}^{0}}{2} \frac{e}{m \omega_{ \pm}} \frac{\omega_{p} \omega_{s}}{\omega_{e}^{4} \omega_{ \pm}} \frac{\left(1-n_{p}^{2}\right)\left(1-n_{s}^{2}\right)}{1-n_{ \pm}^{2}}\left\{\frac{j \vartheta_{0, \pm}}{Q_{0, \pm}^{2}+1} \frac{1+n_{0, \pm}}{n_{ \pm \pm}+n_{0, \pm}}+\frac{j Q_{x, \pm}}{Q_{x, \pm}^{2}+1} \frac{1+n_{x, \pm}}{n_{ \pm}+n_{x, \pm}}\right\} \xi^{ \pm} e^{j \omega_{ \pm} z / c_{0}},
\end{aligned}
$$

where it is to be remembered, that $Q_{0, \pm} Q_{x, \pm}=-1$. 
We notice from (3.49) and (3.50) that only two resonances remain (compare the similar situation in section 1), viz,

or

$$
\left.\begin{array}{l}
n_{0, \pm}=-n_{ \pm} \text {and } n_{x, \pm}=-n_{ \pm}, \\
-\left(\omega_{s} \pm \omega_{p}\right) n_{0, \pm}=\omega_{s} n_{s} \pm \omega_{p} n_{p}, \\
-\left(\omega_{s} \pm \omega_{p}\right) n_{x, \pm}=\omega_{s} n_{s} \pm \omega_{p} n_{p}
\end{array}\right\} .
$$

It is also important to note that these resonances by (3.46) correspond to the correct signs for $n_{0, \pm}$ and $n_{x, \pm}$, and are thus physically possible, if $\left|n_{0, \pm}\right|=\left|\left(\omega_{s} n_{s} \pm \omega_{p} n_{p}\right) /\left(\omega_{s} \pm \omega_{p}\right)\right|$, or if $\left|n_{x, \pm}\right|$ $=\left|\left(\omega_{s} n_{s} \pm \omega_{p} n_{p}\right) /\left(\omega_{s} \pm \omega_{p}\right)\right|$.

The possibilities to obtain these resonances are considerable since $n_{0, \pm}, n_{x, \pm}, n_{s}$ and $n_{p}$ may vary within wide limits in the magneto-ionic medium. The degenerate case $\omega_{+}=2 \omega_{s}$, which is of particular interest if one wishes to study the possibilities of obtaining second harmonic radiation or second harmonic echoes $\left(E_{p, y}^{0}=E_{s, y}^{0}=\right.$ amplitude of higher power primary wave, $\omega_{p}=\omega_{s}$, etc.; $\xi^{+}$assumes a simpler form) has been investigated in detail by the author [Rydbeck, 1962].

The second degenerate case, $\omega_{-}=-\omega_{s}$ (which leads to the same resonance conditions, although $\xi^{-}$is different from $\xi^{+}$of the previous case) is of particular interest, if one wishes to study the possibilities of degenerate parametric amplification in the magneto-ionic medium.

Finally, let us return to the influence of the resonances of the driving forces, a situation that is somewhat different when we discuss the magnitude of $E_{y}$, instead of $v_{y}^{(2)}$.

1. Fourth reflection resonance.

Assume that $\left|n_{p}\right| \rightarrow \infty$. We then find from (3.32) that

i.e.,

$$
\xi^{ \pm} \propto\left(1-n_{ \pm}^{2}\right) n_{p}
$$

$$
\left|E_{y}\right|_{\text {(medium I) }} \propto\left|n_{p}^{3}\left\{\frac{1+n_{0, \pm}}{n_{ \pm}+n_{0, \pm}}+\frac{1+n_{x, \pm}}{n_{ \pm \pm}+n_{x, \pm}}\right\}\right|,
$$

which demonstrates that the influence of the driving force resonance is extremely strong. This appears to be the most interesting nonlinear resonance in the magneto-ionic medium.

2. Plasma resonance.

$$
X_{p} \rightarrow 1 ; n_{0, p} \rightarrow 0, n_{x, p} \rightarrow 1 \text {. }
$$

a. $n_{p}=n_{x, p}$. One finds that

$$
\left|E_{y}\right|_{(\text {medium I })} \propto \frac{1-n_{s}^{2}}{1-n_{ \pm}^{2}}\left\{\frac{1+n_{0, \pm}}{n_{ \pm}+n_{0, \pm}}+\frac{1+n_{x, \pm}}{n_{ \pm}+n_{x, \pm}}\right\}
$$

In contrast to what might be expected, this driving force resonance produces no enhanced effects.

b. $N_{p}=n_{0, p}$. One now finds that

$$
\left|E_{y}\right|_{\text {(medium II })} \propto \frac{1-n_{s}^{2}}{1-n_{ \pm}^{2}} \frac{1}{1-X_{p}}\left\{\frac{1+n_{0, \pm}}{n_{ \pm}+n_{0, \pm}}+\frac{1+n_{x, \pm}}{n_{ \pm}+n_{x, \pm}}\right\}
$$

i.e., the effect of the driving force resonance is very profound. A more detailed study of sum and difference frequency generation caused by this resonance will appear in the final section 5 of this communication.

A further analysis of the traveling wave resonances, (3.51), interesting as it may be, is outside the scope and aim of the present communication. That interesting phenomena, applicable to topside sounder ionogram interpretations, can be found is likely. 


\section{Generation of Natural Sum and Difference Frequency Waves in a Slowly Inhomogeneous Magneto-Ionic Medium}

In order to analyze the resonance radiation of sum and difference frequency magneto-ionic modes, we make use of (3.36), which by (3.10) for our present purposes can be written

$$
\begin{aligned}
\left(\frac{d^{2}}{d z^{2}}+k_{0, \pm}^{2}\right)\left(\frac{d^{2}}{d z^{2}}+k_{x, \pm}^{2} E_{y}^{ \pm}\right) \simeq-\frac{1}{c_{0}^{4} b_{ \pm}} \frac{E_{s . y}^{0} E_{p, y}^{0}}{2} \frac{\tau_{ \pm}}{\tau_{s} \tau_{p}} \omega_{ \pm}^{5}\left(1-X_{ \pm}\right) & \\
& \times\left(1-X_{ \pm}-n_{ \pm}^{2}\right) \xi^{ \pm}=\psi^{ \pm} e^{j\left\{\omega_{ \pm} t-\int_{z_{a}}^{2}\left(k_{s} \pm k_{p}\right) d z\right\}}
\end{aligned}
$$

For the slowly inhomogeneous medium the solution to (4.1) now can be written

$$
\begin{aligned}
E_{y}^{ \pm} \approx-\frac{j}{2} & \frac{\psi^{ \pm} e^{+j \omega_{ \pm} t}}{k_{0, \pm}^{2}-k_{x, \pm}^{2}}\left\{\frac{1}{k_{x, \pm}}\left(e^{+j \int_{z_{a}}^{z} k_{x} \pm d z} \int_{z_{1}}^{z} e^{-j \int_{z_{a}}^{z}\left(k_{s} \pm k_{p}+k_{x}, \pm\right) d z} d z-e^{-j \int_{z_{a}}^{z} k_{x}, \pm d z} \int_{z_{2}}^{z} e^{-j \int_{x_{a}}^{a}\left(k_{s} \pm k_{p}-k_{x} \pm\right) d z} d z\right)\right. \\
& \left.-\frac{1}{k_{0, \pm}}\left(e^{+j \int_{z_{a}}^{z} k_{0, \pm} \pm d z} \int_{z_{3}}^{z} e^{-j \int_{z_{a}}^{a}\left(k_{s} \pm k_{p}+k_{0, \pm}\right) d z} d z-e^{-j \int_{z_{a}}^{z} k_{0} \pm d z} \int_{z_{4}}^{z} e^{-j \int_{z_{a}}^{z}\left(k_{s} \pm k_{p}-k_{0, \pm}\right) d z d z}\right)\right\} . \quad(4.1 \mathbf{a})
\end{aligned}
$$

If we assume that resonance takes place at some specified level $z_{0}$, where, for example

$$
k_{x, \pm}=-\left(k_{s} \pm k_{p}\right),
$$

and conditions are such (see section 2) that a forward extraordinary magneto-ionic mode is generated, we obtain

$\underline{z<z_{0}}\left(W^{2}>>1\right)$

$$
E_{y}^{ \pm} \approx \frac{\psi^{ \pm} e^{j\left(\omega_{ \pm} t-\int_{z_{a}}^{z}\left(k_{s} \pm k_{p}\right) d z\right)}}{\left\{k_{0, \pm}^{2}-\left(k_{s} \pm k_{p}\right)\right\}\left\{k_{x, \pm}^{2}-\left(k_{s} \pm k_{p}\right)^{2}\right\}},
$$

$z>z_{0}\left(W^{2}>>1\right)$

$$
E_{y}^{ \pm} \approx \psi^{ \pm} e^{j\left(\omega_{ \pm} t-\int_{z_{a}}^{\left.z_{0}\left(k_{s} \pm k_{p}\right) d z\right)}\right.}\left[\frac{e^{-j \int_{z_{0}}^{z}\left(k_{s} \pm k_{p}\right) d z}}{\left\{k_{0, \pm}^{2}-\left(k_{s} \pm k_{p}\right)^{2}\right\}\left\{k_{x, \pm}^{2}-\left(k_{s} \pm k_{p}\right)^{2}\right\}}-\frac{\left.e^{+j\left(\int_{z_{0}}^{z} k_{x, \pm} d z+\pi / 4\right.}\right)}{\left(k_{0, \pm}^{2}-k_{x, \pm}^{2}\right) 2 k_{x, \pm}} \cdot \sqrt{\frac{\pi}{2 \mathcal{H}(}}\right] .
$$

[For definition of $\mathcal{H}$ see (2.9).]

Although the amplitude coefficients are much more complicated than in the isotropic case, dealt with in section 2 , the radiation feature is essentially the same. Furthermore it should be pointed out, see (3.32), that the pump wave, for example, does not have to be of extraordinary kind in order to generate an extraordinary natural mode. This is a typical feature of the nonlinear propagation theory.

In conclusion a few words should be said about the ray paths. Since we are dealing with an anisotropic medium these will deviate from the phase paths. In order to make the discussion more general, we assume arbitrary incidence, and noting the direct similarity between the coupling integrals of (2.5) and (4.2), write the phase of the downcoming difference frequency wave as follows,

$$
\phi_{0,-1}=\int_{z_{a}}^{z_{0}}\left(k_{s, z}-k_{p, z}-k_{-1, z}\right) d z+\frac{1}{c_{0}}\left(\omega_{s} \sin \varphi_{s, 0}-\omega_{p} \sin \varphi_{p, 0}\right)\left(x_{2}-x_{1}\right),
$$

where $x_{1}$ and $x_{2}$ are the ray positions corresponding to $z_{a}$, i.e., $x_{2}$ for the downcoming difference frequency wave [note: $\varphi_{s, 0}=\left(\varphi_{s}\right)_{N_{\ell}=0}$, etc.]. 
If the signal wave carries the information, for example in pulses, the position of the downcoming difference frequency wave is determined by the condition that

and by

$$
\frac{\partial \phi_{0,-1}}{\partial \varphi_{s, 0}}=0
$$

$$
\frac{\partial \phi_{0,-1}}{\partial \varphi_{p, 0}}=0
$$

if the pump wave carries the information. The ray direction of the associated wave,

$$
\exp \left[j\left\{\omega_{ \pm} t-\left(k_{s, x} \pm k_{p, x}\right) x-\int^{z}\left(k_{s, z} \pm k_{p, z}\right) d z\right\}\right]
$$

thus has the ray direction of the signal wave or the pump wave, if the former or the latter carries the information. This is another characteristic feature of the nonlinear propagation theory.

Since

etc., and

$$
n_{s, z}=\sqrt{n_{s}^{2}-\sin ^{2} \varphi_{s, 0}}
$$

we obtain

$$
\sin \varphi_{-1,0}=\frac{1}{\omega_{-}}\left(\omega_{s} \sin \varphi_{s, 0}-\omega_{p} \sin \varphi_{p, 0}\right),
$$

$$
\begin{aligned}
& \frac{\partial \phi_{0,-1}}{\partial \varphi_{s, 0}}=-\frac{\omega_{s}}{c_{0}} \cos \varphi_{s, 0} \\
& {\left[\int_{z_{a}}^{z_{0}}\{\underbrace{\left\{\tan \varphi_{s}+\frac{1}{\cos ^{2} \varphi_{s}}\left(\frac{1}{n_{s}} \frac{d n_{s}}{d \theta}\right)_{\varphi_{s}}+\tan \varphi_{-1}+\frac{1}{\cos ^{2} \varphi_{-1}}\left(\frac{1}{n_{-}} \frac{d n_{-}}{d \theta}\right)_{\varphi-1}\right.}_{\mu_{-}}\} d z\right.} \\
&\left.-\left(x_{2}-x_{1}\right)+\left(\frac{2 k_{-1,2}}{\mathscr{H}}\right)_{z_{0}} \mu_{-}\right] .
\end{aligned}
$$

For a very slowly varying medium $x_{2}-x_{1}$ thus approximately becomes [compare (2.14)]

$$
x_{2}-x_{1} \simeq\left\{z_{0}-z_{a}+\left(\frac{2 k_{-1, z}}{\mathscr{H}}\right)_{z_{0}}\right\} \mu_{-} .
$$

Due to the "height dispersion" the ray path of the downcoming difference frequency wave is deformed, as if the frequency shift reflection took place at the level $z_{0}+\left(\frac{2 k_{-1, z}}{\mathcal{H}}\right)$, instead of at $z_{0}$. For this change the wave normal paths in figure 3 have not been corrected.

Returning to our present case of magneto-ionic vertical incidence $\left(\varphi_{s, 0}=0=\varphi_{-1,0}\right)$, we obtain

$$
x_{2}-x_{1} \cong\left\{z_{0}-z_{a}+\left(\frac{2 k_{-1, z}}{\mathcal{H}}\right)_{z_{0}}\right\}\left\{\left(\frac{1}{n_{s}} \frac{d n_{s}}{d \theta}\right)+\left(\frac{1}{n_{-}} \frac{d n_{-}}{d \theta}\right)\right\} \cdot \varphi_{\varphi_{s, 0}=0=\varphi_{p, 0}}
$$

The difference frequency wave therefore does not come down where the signal wave enters, if

Since

$$
\frac{1}{n_{s}} \frac{d n_{s}}{d \theta}+\frac{1}{n_{-}} \frac{d n_{-}}{d \theta} \neq 0
$$

$$
\left(\frac{1}{n_{s}} \frac{d n_{s}}{d \theta}\right)_{\left(\begin{array}{l}
0 \\
x
\end{array}\right)}=\mp Y_{T, s} Y_{L, s}\left(1-n_{s}^{2}\left(\begin{array}{l}
0 \\
x
\end{array}\right)\right) \frac{1}{\sqrt{Y_{T, s}^{4}-4\left(1-X_{s}\right)^{2} Y_{L, s}^{2}}}
$$


$\theta$ would have to be equal to $0^{\circ}$ or $90^{\circ}$ for $\mu_{-}$safely to be equal to zero. Since the nonlinear driving terms disappear if $\theta=0^{\circ}$ (unless we consider second order quantities in the high power pump field), we have to select $\theta=90^{\circ}$, i.e., transverse magneto-ionic propagation, in order to obtain reliable traveling wave resonance effects recorded at the same transmitting and receiving site (for example in a topside sounder).

\section{Sum and Difference Frequency Generation When the Nonlinear Driving Forces Have a Resonance}

For the sake of simplicity we limit ourselves to one resonance only and assume that the signal wave experiences longitudinal plasma resonance at some specified level $z=z_{0}$. If $H$ is the scale height of the electron density, we write accordingly

$$
X_{s}=1+\frac{z-z_{0}}{H} \cdot \quad\left\{\left(\frac{z-z_{0}}{H}\right)^{2} \ll 1\right\}
$$

We now have to include (a modest) collision frequency $\nu_{s}$, and replace

where

$$
\alpha_{s}=Y_{T, s} /\left(1-X_{s}\right) \text { by } \alpha_{s}, \nu=Y_{T . s} /\left(1-X_{s}-j \delta_{s}\right),
$$

$$
\delta_{s}=\nu_{s} / \omega_{s}
$$

If the signal wave is of ordinary type, $n_{s}=n_{s, 0}$ (see $2 \mathrm{~b}$ of sec. 3 ), $\xi^{ \pm}$near and at the resonance level can be written

$$
\begin{aligned}
\xi^{ \pm} \cong \omega_{ \pm}^{2}\left(1-n_{ \pm}^{2}\right) \frac{Y_{T, s}}{1-X_{s}-j \nu_{s}} \frac{\omega_{p}}{c_{0}}\left\{\left(1-\frac{X_{p}}{1-n_{p}^{2}}\right)\left(1 \pm Q_{ \pm} Q_{p}\right) \pm \frac{Y_{T, p}^{2}}{1-X_{p}}\right. & \\
& \left.\left.\times\left(n_{ \pm}+n_{p} X_{ \pm}\right) e^{j\left\{\omega \pm t-\int_{z_{a}}^{2}\left(k_{s \pm} k_{p}\right) d z\right\}}\right\}, \quad X_{p} \neq 1\right)
\end{aligned}
$$

where $k_{s} \cong 0$. We have (naturally) neglected triple split coupling of the signal wave and likewise have assumed it to be of practically constant amplitude through the important part of the resonance region, a helpful but somewhat crude approximation.

Noticing that $b_{ \pm}$can be written

relation (4.1) yields

$$
b_{ \pm}=\omega_{ \pm}^{4}\left(1-X_{ \pm}\right) X_{ \pm}^{2} \frac{1}{\left(1-n_{0, \pm}^{2}\right)\left(1-n_{x, \pm}^{2}\right)}
$$

$$
\left(\frac{d^{2}}{d z^{2}}+k_{0, \pm}^{2}\right)\left(\frac{d^{2}}{d z^{2}}+k_{x, \pm}^{2}\right) E_{y}^{ \pm} \cong \frac{\psi^{ \pm}}{1-X_{s}-j \delta_{s}} e^{j\left\{\omega_{ \pm} t-\int_{z_{a}}^{z}\left(k_{s} \pm k_{p}\right) d z\right\}},
$$

where

$$
\begin{aligned}
\psi^{ \pm}=-E_{s, y}^{0} \frac{v_{p, y, \pm}^{0}}{2}\left(1-n_{p}^{2}\right)\left(1-n_{s}^{2}\right) & \left(1-n_{0, \pm}^{2}\right)\left(1-n_{x, \pm}^{2}\right) \frac{1}{X_{ \pm}} \frac{\omega_{p}^{2} \omega_{s} \omega_{ \pm}}{c_{0}^{4}}\left(1-X_{ \pm}-n_{ \pm}^{2}\right) \\
& \times Y_{T, s}\left\{\left(1-\frac{X_{p}}{1-n_{p}^{2}}\right)\left(1 \pm Q_{ \pm} Q_{p}\right) \pm \frac{Y_{T, p}^{2}}{1-X_{p}}\left(n_{ \pm}+n_{p} X_{ \pm}\right)\right\},
\end{aligned}
$$

and

$$
v_{p, y, \pm}^{0}=E_{p, y}^{0} e / m \omega_{ \pm},
$$

i.e., the a-c electron velocity that the pump wave would generate, in an isotropic medium.

Since the evaluation of ordinary and extraordinary natural sum and difference frequency waves follows the same procedure, we take the ordinary sum and difference frequency waves 
as characteristic examples. By (4.1) we obtain approximately

$$
E_{y_{(0)}}^{+}=+\frac{j}{2} \frac{\psi^{+} e^{j \omega+t}}{k_{0,+}^{2}-k_{x,+}^{2}} \frac{1}{k_{0,+}} e^{+j \int_{z_{a}}^{z} k_{0,+d}} \underbrace{\int_{+\infty}^{z} e^{-j \int_{z_{a}}^{z}\left(k_{s}+k_{p}+k_{0,+}\right) d z} \frac{d z}{1-X_{s}-j \delta_{s}}}_{H \mathscr{\mathcal { F }}_{+} e^{-\int_{z_{a}}^{z_{0}\left(k_{s}+k_{p}+k_{0,+}\right) d z}}},
$$

where it has to be remembered that $k_{0,+}$ must be positive for a downcoming wave. The coupling integral, $\mathscr{F}_{+}$, now becomes (since $k_{s} \approx 0$ )

where

$$
\mathscr{f}_{+}=\mathscr{f}^{*}\left(y, \sigma_{+}\right)=\int_{-\infty}^{y} \frac{e^{+j y}}{y-j \sigma_{+}} d y
$$

One finds that

$$
\sigma=\delta_{s} H\left(k_{p}+k_{0,+}\right) \text { and } y=\left(z_{0}-z\right)\left(k_{p}+k_{0,+}\right) \text {. }
$$

$$
\mathcal{f}^{*}\left(+\infty, \sigma_{+}\right)=\left\{\begin{array}{l}
j 2 \pi e^{-\sigma_{+}}, \sigma_{+}>0 \\
j \pi, \quad \sigma_{+}=0 \\
0, \quad \sigma_{+}<0
\end{array}\right.
$$

Assuming $k_{p}$ to be positive (pump wave running in positive $z$-direction), we thus find that a sum frequency "echo" is obtained only if the electron density gradient is positive. This is in accordance with the physical situation, since the signal wave runs in direction of increasing $X_{s}$.

In order to demonstrate how the "reflected" difference frequency wave is being built up in the driving force resonance region, we have in figure 4 presented the amplitude of the coupling integral as a function of $y$. One notes the (infinite) plasma resonance for $\sigma=0$, and the moderate field perturbation in the resonance region, when $H<0$.

If we assume that the pump frequency is larger than $2 \omega_{s}$, so that a difference frequency wave in principle could propagate away from the resonance region, the coupling integral becomes

where

$$
\mathscr{J}_{-}=\mathscr{f}\left(y, \sigma_{-}\right)=\int_{-\infty}^{y} \frac{e^{-j y}}{y+j \sigma_{-}} d y
$$

$$
\sigma_{-}=\delta_{s} H\left(k_{p}+k_{0,-}\right) \text {, and } y=\left(z_{0}-z\right)\left(k_{p}+k_{0,-}\right) .
$$

It appears from (5.10) that

$$
\mathcal{f}\left(+\infty, \sigma_{-}\right)=\left\{\begin{array}{cc}
-j \pi e^{-\sigma}, & \sigma_{-}>0 \\
-j \pi, & \sigma_{-}=0 \\
0, & \sigma_{-}<0
\end{array}\right.
$$

Figure 4. Depicting buildup of downcoming sum frequency wave.

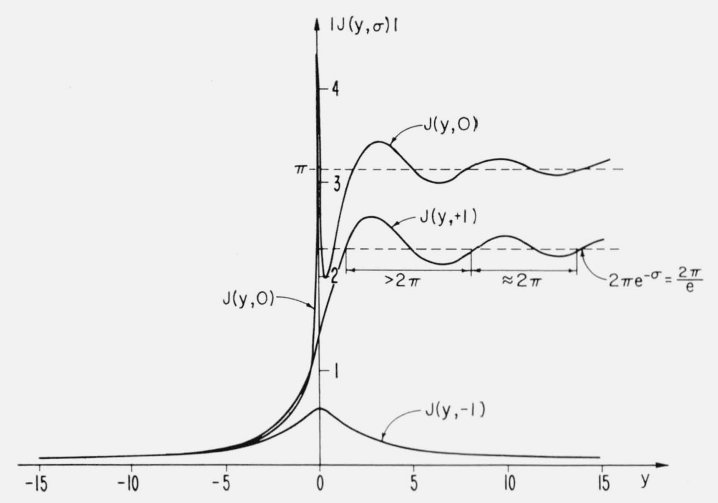


Thus, also in the difference frequency case $\left(\omega_{p}>2 \omega_{s}\right)$ a positive electron density gradient is required for a downcoming wave to be generated.

For the ordinary forward sum frequency wave the coupling integral becomes

$$
\begin{gathered}
\mathscr{J}_{+}=\mathscr{f}(y, \sigma) \text {, where } \begin{cases}\sigma=\delta_{s} H\left(k_{p}-k_{0,+}\right) & \left(k_{p}>k_{0,+}\right) \\
y=\left(z-z_{0}\right)\left(k_{p}-k_{0,+}\right) & \end{cases} \\
\mathscr{f}_{+}=-\mathscr{f}^{*}(y, \sigma), \text { where }\left\{\begin{array}{ll}
\sigma=\delta_{s} H\left(k_{0,+}-k_{p}\right) & \left(k_{p}<k_{0,+}\right) \\
y=\left(z-z_{0}\right)\left(k_{0,+}-k_{p}\right) &
\end{array}\right\} .
\end{gathered}
$$

Thus, even the generation of a forward wave requires a positive electron density gradient.

The downcoming sum frequency wave, at the level $z=z_{a}$, finally can be written

where

$$
E_{y,(0)}^{+} \cong R_{0,+1}^{(0)} E_{s, y}^{0}
$$

Since

$$
\begin{aligned}
R_{0,+1}^{(0)}= & -\frac{1}{2} \frac{v_{p, y}^{0}}{c_{0}}(\underbrace{\pi k_{0,+} H e^{-\delta_{s} H \mid k} p^{+k_{0,+}}}_{\rho})_{z=z_{0}} e^{j\left(\omega_{+} t-\int_{z_{a}}^{z_{0}}\left(k_{s}+k_{p}+k_{0,+} ; d z\right.\right.} \\
\times & \times\left[\frac{\omega_{p}^{2} \omega_{s}}{\omega_{e}^{2} \omega_{+}} \frac{\left(1-n_{p}^{2}\right)\left(1-n_{0,+}^{2}\right)\left(1-n_{x,+}^{2}\right)}{\left(n_{0,+}^{2}-n_{x,+}^{2}\right) n_{0,+}^{2}}\left(1-X_{+}-n_{+}^{2}\right)\right. \\
& \left.\times Y_{T, s}\left\{\left(1-\frac{X_{p}}{1-n_{p}^{2}}\right)\left(1-Q_{+} Q_{p}\right)-\frac{Y_{T, p}^{2}}{1-X_{p}}\left(n_{+}+X_{+} n_{p}\right)\right\}\right]_{z=z_{0}} .
\end{aligned}
$$

$$
\rho=\rho_{\max }=\frac{\omega_{s}}{\nu_{s}} \frac{\pi}{e} \frac{k_{0,+}}{\left|k_{p}+k_{0,+}\right|}, \text { for } H=\frac{\omega_{s}}{\nu_{s}} \frac{1}{\left|k_{p}+k_{0,+}\right|},
$$

an optimum that should be attainable at some levels in the ionosphere, the order of magnitude of $\left|R_{0,+1}^{(0)}\right|$ is $v_{p, y}^{0} \omega_{c} / c_{0} \nu_{s}$, if $\omega_{p}$ is moderately larger than $2 \omega_{s}$.

If we assume the pump wave (the high power wave) to be pulsed, and the signal wave to be continuous, the sum frequency "echo" delay becomes

$$
\tau_{+}^{(0)}=\frac{\partial}{\partial \omega_{p}} \int_{z_{a}}^{z_{0}}\left(k_{s}+k_{p}+k_{0,+}\right) d z \cong \frac{2}{c_{0}}\left\{z_{0}-z_{a}+H\left(\frac{2 \omega_{p}}{\omega_{s}}+1\right)\right\}
$$

if $\omega_{p}$ is so large that $\left(n_{p}\right)_{z_{0}} \cong 1$. For the difference frequency "echo" delay we similarly obtain

$$
\tau_{-}^{(0)} \cong \frac{2}{c_{0}}\left\{z_{0}-z_{a}+H\left(\frac{2 \omega_{p}}{\omega_{s}}-1\right)\right\},
$$

provided that $\omega_{p}-\omega_{s}$ is so great, that $\left(n_{0,-}\right)_{z_{0}} \cong 1$. These relations yield

and

$$
H=\frac{c_{0}}{4}\left(\tau_{+}^{(0)}-\tau_{-}^{(0)}\right),
$$

$$
z_{0}=z_{a}+\frac{c_{0}}{4}\left\{\tau_{+}^{(0)}+\tau_{-}^{(0)}-\left(\tau_{+}^{(0)}-\tau_{-}^{(0)}\right) \frac{2 \omega_{p}}{\omega_{s}}\right\}
$$

By sum and different frequency pulse "echo" measurement of this kind it is thus in principle possible (if $\omega_{p}-\omega_{s}$ is large enough) to obtain not only the true ordinary reflection height, $z_{0}\left(X_{s}=1\right)$, but also the electron density gradient, $N_{e} / H$, at the same. Since the pulse 
measurements have to be done at relatively high frequencies, for example $f_{s}=30 \mathrm{Mc} / \mathrm{s}, \tau_{+}^{(0)}$ and $\tau_{-}^{(0)}$ can be determined with much greater accuracy than at the unshifted signal frequency $\omega_{s}$. Another advantage is the fact that $\omega_{p}$, if it is large enough, can remain unchanged, while $\omega_{s}$ is swept through the probing frequency range of interest.

The immediate question one asks oneself is the following: Is it practically possible to perform sum and difference frequency pulse echo measurements of the type just discussed, using ground based equipment? If $\omega_{p}^{2}>>\omega_{0}^{2}$, the amplitude of $R_{0,+1}^{(0)}$ by (5.16) approximately becomes

where

$$
\left|R_{0,+1}\right| \approx \frac{1}{4} \frac{v_{p, y}^{0}}{c_{0}} \frac{\omega_{s}}{\nu_{s}} \frac{\pi}{e} \underbrace{u e^{1-u}}_{\mu} \frac{\omega_{0}^{4}}{\left(\omega_{p}+\omega_{s}\right)^{3} \omega_{p}} Y_{T, s},
$$

$$
u=\frac{\nu_{s}}{\omega_{s}} H\left|k_{p}+k_{0,+}\right|
$$

If we assume $u$ to lie in the range 0.08 to 4 , i.e., within a $u$-ratio of $50, \mu \geq 0.20\left(\mu_{\max }=1\right)$, and $\omega_{p} / 2 \pi=30 \mathrm{Mc} / \mathrm{s}, \omega_{s} / 2 \pi=5 \mathrm{Mc} / \mathrm{s}$, we obtain

$$
\left|R_{0,+1}\right| \approx \frac{2 v_{p, y}^{0}}{c_{0}} \frac{f_{s}}{\nu_{s}} 10^{-4} Y_{T, s}
$$

which, with $\nu_{s}=10^{3}$, yields $\left|R_{0,+1}\right| \approx \frac{v_{p, y}^{0}}{c_{0}} Y_{T, s}$. If the ground based pump transmitter has an effective radiation aperture $A$, and the ionospheric absorption is neglected at the pump frequency, $v_{p, y}^{0}$ becomes

$$
v_{p, y}^{0} \approx \frac{e}{m} \frac{1}{\pi c_{0} r} \sqrt{\frac{A Z_{0} P_{0}}{2}}, \quad\left(Z_{0}=\sqrt{\frac{\mu_{0}}{\epsilon_{0}}}\right)
$$

where $P_{0}$ is the transmitter power and $r$ the distance from the ground to the $z_{0}-$ level. Considering the fact that the returning sum (or difference) frequency travels practically without absorption and that the atmospheric noise level at the sum (or difference) frequency is very low, compared to at $f_{s}=5 \mathrm{Mc} / \mathrm{s}$, we may assume that a $v_{p, y}^{0} / c_{0}$-ratio of $10^{-5}$ would yield a detectable echo at the ground (provided the regular ionosonde at $5 \mathrm{Mc} / \mathrm{s}$ is powerful enough). With an effective antenna area of $10^{4} \mathrm{~m}^{2}$, and $r_{0}=300 \mathrm{~km}, P_{0}$ becomes $5 \mathrm{MW}$. It thus seems to lie within the possibilities of present day techniques to perform ionospheric sum and difference frequency measurements as outlined. Beyond doubt practical experiments of this type would be very interesting and probably also rewarding.

The research reported in this communication was partly done at the Ionosphere Research Laboratory (IRL), Pennsylvania State University, and partly at the Research Laboratory of Electronics, Gothenburg, Sweden. The author is much indebted to the IRL for its hospitality. The Air Force Cambridge Research Laboratories, Bedford, supported two thirds of the research under contract $\mathrm{AF} 61(052)-451$ with the Research Laboratory of Electronics, through the European Office of Aero Space Research.

\section{References}

Rydbeck, O.E.H., and B. Agdur (1954), The propagation of electronic space charge waves in periodic structures, Transactions of Chalmers University of Technology, No. 138.

Rydbeck, O.E.H. (1961), Dynamic nonlinear wave propagation in ionized media (DNWP), I, The isotropic medium, Research Report No. 27, Research Laboratory of Electronics (RLE), Chalmers University of Technology (CUT), Gothenburg, Sweden.

Rydbeck, O. E. H. (1962), The magneto-ionic medium (DNWP) II, Research Report No. 28, RLE.

Rydbeck, O. E. H. (1963), Electromagnetic nonlinear wave interaction and reflection from a plane ionized medium (DNWP) III, Research Report No. 29, RLE.

Rydbeck, O. E. H. (1964), Dynamic non-linear magneto-ionic wave propagation, Arkiv för Geofysik (Trans. Roy. Swed. Acad. Sci.) to be published. 


\section{Additional Related References}

Wilhelmsson, K. H. B. (1962), Reflection of electromagnetic waves from an ionized stream in the presence of a high power primary wave (DNWP) V, Research Report No. 31, RLE.

Wilhemsson, K. H. B. (1962), Scattering of electromagnetic waves by an anisotropic medium with a harmonically time-varying dielectric tensor, Research Report No. 32, RLE.

Thomasson, A. R. (1963), Reflection of electromagnetic waves from a dielectric plane layer varying periodically in space and time (DNWP) VIII, Research Report No. 34, RLE.

Thomasson, A. R. (1964), Microscopic treatment of the interaction between a plane electromagnetic wave and an oscillating medium (DNWP) XIII, Research Report No. 44, RLE.

Copies of these reports can be obtained from the Librarian, Research Laboratory of Electronics, Chalmers University of Technology, Gibraltargatan 5G, Gothenburg S, Sweden.

(Paper 69D1-447) 